TRANSACTIONS OF THE

AMERICAN MATHEMATICAL SOCIETY

Volume 353, Number 12, Pages 4925-4948

S 0002-9947(01)02740-4

Article electronically published on April 18, 2001

\title{
CONDITIONS IMPOSED BY TACNODES AND CUSPS
}

\author{
JOAQUIM ROÉ
}

\begin{abstract}
The study of linear systems of algebraic plane curves with fixed imposed singularities is a classical subject which has recently experienced important progress. The Horace method introduced by A. Hirschowitz has been successfully exploited to prove many $H^{1}$-vanishing theorems, even in higher dimension. Other specialization techniques, which include degenerations of the plane, are due to Z. Ran and C. Ciliberto and R. Miranda. G. M. Greuel, C. Lossen and E. Shustin use a local specialization procedure together with the Horace method to give the first asymptotically proper general existence criterion for singular curves of low degree. In this paper we develop a specialization method which allows us to compute the dimension of several linear systems as well as to substantially improve the bounds given by Greuel, Lossen and Shustin for curves with tacnodes and cusps.
\end{abstract}

\section{INTRODUCTION}

A closed subscheme $Z \subset \mathbb{P}^{2}$ is said to have maximal rank in degree $d$ if the canonical map $H^{0}\left(\mathbb{P}^{2}, \varnothing_{\mathbb{P}^{2}}(d)\right) \rightarrow H^{0}\left(Z, \varnothing_{Z}(d)\right)$ has maximal rank (cf. [23]). If $Z$ is a zero-dimensional scheme of length $N$, this means that either there are no curves of degree $d$ containing $Z$ or $Z$ imposes $N$ independent linear conditions to curves of degree $d$. If $Z$ has maximal rank in all degrees, then we simply say that it has maximal rank.

For some classes of zero-dimensional schemes, it is known that general members have maximal rank. For example, a general union of double points has maximal rank. In many other cases, however, a maximal rank statement has been conjectured only (cf. [18], 24], 28]). We shall consider schemes $Z=Z_{1} \cup Z_{2} \cup \ldots \cup Z_{\rho}$ where $Z_{i}$ are unibranched cluster schemes all whose points but one, whether proper or infinitely near, are taken with multiplicity $\leq 2$. In Theorem 4.8 we prove that, under some mild numerical conditions, a scheme $Z$ as above whose points are in general position has maximal rank. This generalizes and unifies a range of previously known results:

- In [23, A. Hirschowitz proves that a general union of distinct points with multiplicity 2 or 3 has maximal rank. Our result generalizes the multiplicity 2 case by allowing infinitely near points, which are not easily dealt with by the Horace method.

Received by the editors July 5, 1999 and, in revised form, April 13, 2000.

1991 Mathematics Subject Classification. Primary 14C20; Secondary 14H20, 14J26, 14H50, $14 \mathrm{C} 05$.

Partially supported by CIRIT 1997FI-00141, CAICYT PB95-0274, and "AGE-Algebraic Geometry in Europe" contract no. ERB940557. 
- M. V. Catalisano and A. Gimigliano in [6] and C. Ciliberto and R. Miranda in [7] prove that a union of general unibranched cluster schemes all whose points have multiplicity one (curvilinear schemes) has maximal rank. Our result generalizes this by allowing multiplicity 2 points.

- In 8, C. Ciliberto and R. Miranda consider quasi-homogeneous schemes, which consist of distinct multiple points, all whose multiplicities but one are taken to be equal. They prove a quite general maximal rank theorem which includes the computation of the superabundant systems (corresponding to multiplicities for which the general scheme does not have maximal rank). In the particular case in which one has a point of multiplicity not bigger than 5 and all other points have multiplicity 2 , we extend their result by allowing infinitely near points.

Other results concerning linear systems with infinitely near base points can be found in the literature, mainly in [17, 20], 14] and [27]. Harbourne's results deal with clusters of points lying on conics and cubics, which are not necessary to us, and the $H^{1}$-vanishing of [14] and [27] is weaker than proving maximal rank.

The maximal rank theorem will allow us to prove the existence of irreducible curves of low degree with tacnodes and higher order cusps. The reasoning is similar to that of [2, 14] or 27]. Theorem 4.12 is much sharper than the result by Greuel, Lossen and Shustin (which nevertheless applies to any kind of plane curve singularities) and also sharper than the one Lossen obtains for tacnodes and cusps (A-singularities), in part because our specialization avoids the use of the Viro method to "glue" the singularities. On the other hand, Barkats' result is a little bit sharper than ours, but it is restricted to nodes and ordinary cusps only.

\section{Preliminaries}

Let $k$ be an algebraically closed field of characteristic zero, $p$ a smooth point of a surface $S$ defined over $k, \varnothing=\varnothing_{S, p}$ the local ring of $p$ on $S, \mathfrak{m}=\mathfrak{m}_{p}$ its maximal ideal.

Consider a sequence of blowing-ups

$$
S_{r} \stackrel{\sigma_{r}}{\longrightarrow} S_{r-1} \longrightarrow \cdots \stackrel{\sigma_{2}}{\longrightarrow} S_{1} \stackrel{\sigma_{1}}{\longrightarrow} S_{0}=S
$$

where $\sigma_{1}$ is the blowing-up of $p$ and for $i>1$ the center of $\sigma_{i}$ is a point $p_{i}$ which lies on the exceptional divisor of $\sigma_{i-1}$. The sequence $K=\left(p_{1}=p, p_{2}, \ldots, p_{r}\right)$ is a cluster with origin at $p$ for which every point is infinitely near to the preceding one; we shall call these clusters unibranched. We write $S_{K}=S_{r}$ and $\pi_{K}: S_{K} \rightarrow S$ the composition of the blowing-ups. Usual facts known for clusters hold in particular for unibranched clusters; we now review some of them, referring the reader to [4], 5] for the proofs.

A point $p_{j}$ is said to be proximate to $p_{i}, j>i$, if and only if it lies on the exceptional divisor of blowing up $p_{i}$ (that is, $j=i+1$ ) or on its strict transform when $j>i+1$. Every point in a unibranched cluster is proximate either to one or to two points, except for $p_{1}$, which is proximate to no one; if $p_{j}$ is proximate to $p_{i}$, then all the points between them are also proximate to $p_{i}$. If $p_{j}$ is proximate to $p_{i}$, then there is a unique point in the exceptional divisor of $\sigma_{j}$ proximate to both $p_{i}$ and $p_{j}$; otherwise there is none. If a point is proximate to two points, it is called a satellite, otherwise it is free. When considering more than one cluster at a time, 
we shall write $p_{i}(K)$ and $S_{i}(K)$ for the $i$ th point of the cluster $K$ and the surface obtained by blowing up the first $i$ points of $K$.

A system of multiplicities for a cluster $K=\left(p_{1}, p_{2}, \ldots, p_{r}\right)$ is a sequence of integers $\mathbf{m}=\left(m_{1}, m_{2}, \ldots, m_{r}\right)$, and a pair $(K, \mathbf{m})$ where $K$ is a cluster and $\mathbf{m}$ a system of multiplicities is called a weighted cluster. A system of multiplicities like

$$
(m, 2,2, \stackrel{i}{.}, 2,1,1, \stackrel{j}{\leftarrow}, 1,0,0, \ldots, 0)
$$

will be designed as $\left(m, 2^{i}, 1^{j}\right)$. Given a weighted cluster, there are an ideal and a zero-dimensional subscheme of $S$ associated to it. Let $E_{i}$ be the pullback (total transform) in $S_{K}$ of the exceptional divisor of blowing up $p_{i}$. Then the ideal sheaf

$$
\mathcal{H}_{K, \mathbf{m}}=\left(\pi_{K}\right)_{*} \varnothing_{S_{K}}\left(-m_{1} E_{1}-m_{2} E_{2}-\cdots-m_{r} E_{r}\right)
$$

is supported at $p$, its stalk at $p$ is a complete $\mathfrak{m}$-primary ideal $H_{K, \mathbf{m}} \subset \varnothing$ and defines a zero-dimensional subscheme $Z_{K, \mathbf{m}}$ of $S$. For $K$ unibranched we call $Z_{K, \mathbf{m}}$ a unibranched cluster scheme. As an aside, note that if $I \subset \varnothing$ is a complete $\mathfrak{m}$ primary ideal, then there is a weighted cluster $(K, \mathbf{m})$ such that $I=H_{K, \mathbf{m}}$, but this cluster does not need to be unibranched.

The same (unibranched) cluster scheme is sometimes defined by different (unibranched) weighted clusters. In this case we shall say that both clusters are equivalent. In order to have a well-defined weighted cluster associated to every cluster scheme, one considers the notion of consistent clusters, which we define next. The proximity inequality at $p_{i}$ is

$$
m_{i} \geq \sum_{p_{j} \text { prox. to } p_{i}} m_{j} .
$$

A weighted cluster $(K, \mathbf{m})$ is consistent if and only if it satisfies the proximity inequalities at all its points. Given a (unibranched) cluster scheme $Z$, there is a unique consistent weighted cluster $(K, \mathbf{m})$ such that $Z=Z_{K, \mathbf{m}}$ and $m_{i}>0$ for all $i$. Furthermore, for a weighted cluster $(K, \mathbf{m})$ nonnecessarily consistent,

$$
\text { length } Z_{K, \mathbf{m}}=\operatorname{dim} \frac{\varnothing}{H_{K, \mathbf{m}}} \leq \sum_{i=1}^{r} \frac{m_{i}\left(m_{i}+1\right)}{2},
$$

with the equality holding if $(K, \mathbf{m})$ is consistent.

Given an arbitrary weighted cluster $(K, \mathbf{m})$ there is a procedure called unloading (see [5, 4], [10, IV.II], or [4]) which gives a new system of multiplicities $\delta(\mathbf{m})=$ $\delta_{K}(\mathbf{m})$ such that $(K, \delta(\mathbf{m}))$ is consistent and equivalent to $(K, \mathbf{m})$. In each step of the procedure, one unloads some amount of multiplicity on a point $p_{i}$ whose proximity inequality is not satisfied, from the points proximate to it. This means that there is an integer $n>0$ such that, increasing the multiplicity of $p_{i}$ by $n$ and decreasing the multiplicity of every point proximate to $p_{i}$ by $n$, the resulting weighted cluster is equivalent to $(K, \mathbf{m})$ and satisfies the proximity inequality at $p_{i}$. In other words, if $\tilde{E}_{i} \subset S_{K}$ is the strict transform of the exceptional divisor of blowing-up $p_{i}, D=-m_{1} E_{1}-m_{2} E_{2}-\cdots-m_{r} E_{r}$ and $\tilde{E}_{i} \cdot D<0$, then one chooses $n$ as the minimal integer with $\tilde{E}_{i} \cdot\left(D-n \tilde{E}_{i}\right) \geq 0$ and replaces $D$ by $D-n \tilde{E}_{i}$. A finite number of unloading steps lead to the desired equivalent consistent cluster $(K, \delta(\mathbf{m}))$.

Let $C \subset S$ be a curve (more generally, a divisor). For any proper or infinitely near point $q$ of $S$ we denote by $e_{q}(C)$ the multiplicity of (the strict transform of) $C$ at $q$. Let $(K, \mathbf{m})$ be a weighted cluster of $r$ points and $i \leq r$. Let $S_{i}$ be the surface 
obtained by blowing up the first $i$ points of $K, \bar{C} \subset S_{i}$ the pullback of $C$ and for $1 \leq j \leq i, E_{j} \subset S_{i}$ the pullback of the exceptional divisor of blowing up $p_{j}(K)$. We define the virtual transform of $C$ in $S_{i}$ relative to the system $\mathbf{m}$ as the divisor

$$
\tilde{C}=\bar{C}-m_{1} E_{1}-m_{2} E_{2}-\cdots-m_{i} E_{i} .
$$

A curve $C$ contains the cluster scheme $Z_{K, \mathbf{m}}$ if and only if its virtual transform in $S_{i-1}$ has multiplicity at least $m_{i}$ at $p_{i}$ for all $i$. Then we say that $C$ goes through $(K, \mathbf{m})$. If $e_{p_{i}}(C)=m_{i}$ for all $i$, then the virtual transform coincides with the strict transform; furthermore, if $\bar{C}$ is a divisor with normal crossings, then we say that $C$ goes sharply through $(K, \mathbf{m})$. In this case, $\pi_{K}$ is an embedded resolution of singularities for $C$, and $(K, \mathbf{m})$ determines the equisingularity class of $C$. There are curves going sharply through $(K, \mathbf{m})$ if and only if $(K, \mathbf{m})$ is consistent, and in this case they are all equisingular.

We define next varieties $Y_{i} \subset X_{i}$ and smooth surjective morphisms $\psi_{i}: X_{i} \rightarrow$ $Y_{i-1}$ of relative dimension 2, as follows: Let $Y_{-1}=\operatorname{Spec} k, X_{0}=S$ and $Y_{0}=\{p\}$, and for $i>0$, let

$$
X_{i} \stackrel{b_{i}}{\longrightarrow} Y_{i-1} \times_{Y_{i-2}} X_{i-1}
$$

be the blowing-up along the diagonal $\Delta\left(Y_{i-1}\right) \subset Y_{i-1} \times_{Y_{i-2}} X_{i-1}$, let $Y_{i} \subset X_{i}$ be the exceptional divisor, and $\psi_{i}$ the composition $\pi_{Y_{i-1}} \circ b_{i}: X_{i} \rightarrow Y_{i-1}$. We define also morphisms $\pi_{i}=\pi_{X_{i-1}} \circ b_{i}$ and $\pi_{i, j}=\pi_{j+1} \circ \cdots \circ \pi_{i-1} \circ \pi_{i}: X_{i} \rightarrow X_{j}$.

Note that by construction the variety $Y_{i}$ is irreducible and smooth, and $\left.\psi_{i}\right|_{Y_{i}}$ : $Y_{i} \rightarrow Y_{i-1}$ is a $\mathbb{P}^{1}$-bundle, therefore $Y_{i}$ is also projective and rational for all $i$. The varieties $X_{i}$ are irreducible, smooth, projective and rational if $S$ is. $Y_{r-1}$ can be identified with the set of all unibranched clusters of $r$ points of $S$ with origin at $p$ (cf. [33], [19, 31]) in such a way that for all $K=\left(p_{1}, p_{2}, \ldots, p_{r}\right) \in Y_{r-1}$, denoting by $j_{K}:\{K\} \rightarrow Y_{r-1}$ the inclusion, one has a pullback diagram

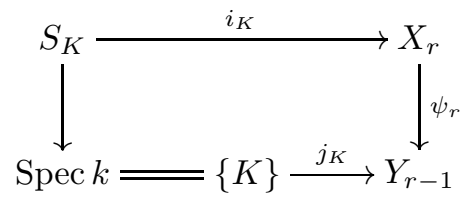

where $i_{K}$ is a closed immersion, and a commutative square

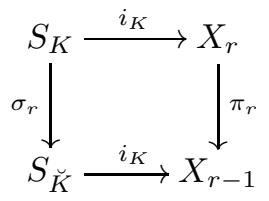

where $\breve{K}=\left(p_{1}, p_{2}, \ldots, p_{r-1}\right)=\psi_{r-1}(K) \in Y_{r-2}$ is the cluster of the first $r-1$ points of $K$, and $\sigma_{r}$ is the blowing-up of $p_{r}(K)$. note that $S_{\breve{K}}=S_{r-1}(K)$ and $i_{\breve{K}}\left(p_{r}(K)\right)=K$.

For every pair of integers $s, t$ such that $r \geq s>t \geq 1$ the subset of $Y_{r-1}$ containing exactly the clusters $K$ with $p_{s}(K)$ proximate to $p_{t}(K)$ is an irreducible smooth closed subvariety $P_{s, t}$ (cf. [33]). There are also open and dense subsets $U_{s, t} \subset P_{s, t}$ which contain all unibranched clusters with $p_{i}(K)$ proximate to $p_{i-1}(K)$ for all $i>1$ and to $p_{t}(K)$ if $s \geq i>t$, and no other proximity relations. We shall write $P_{s}=P_{s, 1}$ and $U_{s}=U_{s, 1}$. We say that a system of multiplicities $\mathbf{m}$ is 


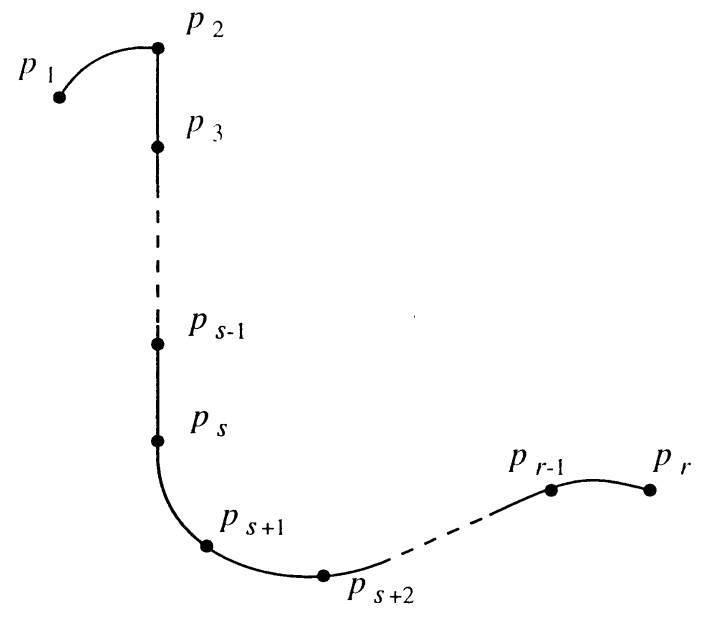

Figure 1. Enriques diagram of a cluster in $U_{s}$

consistent in a subset $W \subset Y_{r}$ when the weighted cluster $(K, \mathbf{m})$ is consistent for all $K \in W$.

Lemma 2.1. Fix a cluster $K=\left(p_{1}, p_{2}, \ldots, p_{r}\right) \in U_{s}$. Let $i, j$ be positive integers such that $r=i+j+1$ and consider the systems of multiplicities

$$
\begin{aligned}
& \mathbf{m}_{-}=\left(m_{1}, 2^{i}, 1^{j}\right), \\
& \mathbf{m}_{+}=\left(m_{1}, 2^{i+1}, 1^{j-1}\right) .
\end{aligned}
$$

Let $I \subset \varnothing$ be an ideal such that

$$
H_{K, \mathbf{m}_{+}} \varsubsetneqq I \varsubsetneqq H_{K, \mathbf{m}_{-}} .
$$

Then there is a point $q \in E_{r}$ such that $I=H_{K_{q}, \mathbf{m}_{0}}$ with $K_{q}=\left(p_{1}, p_{2}, \ldots, p_{r}, q\right)$ and $\mathbf{m}_{0}=\left(m_{1}, 2^{i}, 1^{j+1}\right)$.

Proof. For any $q \in E_{r}$ let $q^{\prime}$ be the unique point that is proximate to $q$ and to $p_{r}$, and consider $K_{q}^{\prime}=\left(p_{1}, p_{2}, \ldots, p_{r}, q, q^{\prime}\right)$ and $\mathbf{m}^{\prime}=\left(m_{1}, 2^{i}, 1^{j+2}\right)$. By performing successive unloading steps on $p_{r}, p_{r-1}, \ldots, p_{i+2}$ we see that $\left(K_{q}^{\prime}, \mathbf{m}^{\prime}\right)$ is equivalent to $\left(K, \mathbf{m}_{+}\right)$and since $\left(K_{q}^{\prime}, \mathbf{m}^{\prime}\right)$ results from $\left(K, \mathbf{m}_{-}\right)$by adding two simple points,

$$
\operatorname{dim} \frac{H_{K, \mathbf{m}_{-}}}{H_{K, \mathbf{m}_{+}}} \leq 2 .
$$

The hypothesis on $I$ implies that the inequality is in fact an equality and

$$
\operatorname{dim} \frac{I}{H_{K, \mathbf{m}_{+}}}=\operatorname{dim} \frac{H_{K, \mathbf{m}_{-}}}{I}=1,
$$

so $I=H_{K, \mathbf{m}_{+}}+(f)$ for some $f \in H_{K, \mathbf{m}_{-}} \backslash H_{K, \mathbf{m}_{+}} \cdot f$ is the equation of a germ of curve $\xi$ which goes through $\left(K, \mathbf{m}_{-}\right)$but not through $\left(K, \mathbf{m}_{+}\right)$, therefore its virtual transform at $p_{r}$ relative to the system of multiplicities $\mathbf{m}_{-}$is smooth, so it has a unique point $q \in E_{r}$. It is clear that $I=H_{K, \mathbf{m}_{+}}+(f) \subseteq H_{K_{q}, \mathbf{m}_{0}}$ and $\operatorname{dim}\left(H_{K_{q}, \mathbf{m}_{0}} / H_{K, \mathbf{m}_{+}}\right) \leq 1$, so $I=H_{K_{q}, \mathbf{m}_{0}}$. 
Proposition 2.2. Let $(K, \mathbf{m})$ be a weighted cluster supported at $p$ and $f \in \varnothing$ the equation of a germ of curve $\xi$ through $p$. Write $e_{i}=e_{p_{i}}(\xi)$. Then the conductor $\left(H_{K, \mathbf{m}}: f\right)$ is $H_{K, \mathbf{m}^{\prime}}$ with

$$
m_{i}^{\prime}=m_{i}-e_{i} .
$$

Proof. The total transform of $\xi$ in $S_{K}$ is

$$
\tilde{\xi}+e_{1} E_{1}+e_{2} E_{2}+\cdots+e_{r} E_{r}
$$

where $\tilde{\xi}$ is the strict transform. Let $g \in \varnothing$ be the equation of a second germ $\eta$. If $g \in H_{K, \mathbf{m}^{\prime}}$, the total transform of $\xi+\eta$ is

$$
\tilde{\xi}+C+\left(\left(m_{1}-e_{1}\right)+e_{1}\right) E_{1}+\left(\left(m_{2}-e_{2}\right)+e_{2}\right) E_{2}+\cdots+\left(\left(m_{r}-e_{r}\right)+e_{r}\right) E_{r}
$$

with $C$ effective, so clearly $f g \in H_{K, \mathbf{m}}$. Conversely, if $f g \in H_{K, \mathbf{m}}$, then the total transform of $\xi+\eta$ is

$$
\tilde{\xi}+C+m_{1} E_{1}+m_{2} E_{2}+\cdots+m_{r} E_{r}
$$

with $C$ effective, so the total transform of $\eta$ must be

$$
C+\left(m_{1}-e_{1}\right) E_{1}+\left(m_{2}-e_{2}\right) E_{2}+\cdots+\left(m_{r}-e_{r}\right) E_{r}
$$

and $g \in H_{K, \mathbf{m}^{\prime}}$.

If $Z \subset S$ is a zero-dimensional scheme defined by the ideal sheaf $\mathcal{I}_{Z / S}$ and $C \subset S$ is a curve, then there is an exact sequence

$$
0 \longrightarrow \mathcal{I}_{Z^{\prime} / S}(-C) \longrightarrow \mathcal{I}_{Z / S} \longrightarrow \mathcal{I}_{(Z \cap C) / C} \longrightarrow 0
$$

where $\mathcal{I}_{(Z \cap C) / C}=\mathcal{I}_{Z / S} \otimes \varnothing_{C}$ and $\mathcal{I}_{Z^{\prime} / S}$ defines a zero-dimensional scheme $Z^{\prime} \subset S$. In the context of the Horace method, it is usually called the residual exact sequence and $Z^{\prime}$ is the residual scheme of $Z$ with respect to $C$ (cf. 23]).

Corollary 2.3. If $Z_{K, \mathbf{m}} \subset S$ is a cluster scheme and $C$ is a curve with $e_{p_{i}}(C)=e_{i}$, then the residual scheme $Z^{\prime}$ of $Z_{K, \mathbf{m}}$ with respect to $C$ is the cluster scheme $Z_{K, \mathbf{m}^{\prime}}$ with $\mathbf{m}^{\prime}$ as in Proposition 2.2

Proposition 2.4. Given a cluster $K=\left(p_{1}, p_{2}, \ldots, p_{r}\right) \in U_{s}$ and a point $q \in E_{r}$, consider $K_{q}=\left(p_{1}, p_{2}, \ldots, p_{r}, q\right)$. Suppose that $r \geq(s-1)(s-2)$. Then for any $q, q^{\prime} \in E_{r}$, each of them proximate only to $p_{r}$, there is an automorphism $\varphi^{*}: \varnothing \rightarrow \varnothing$ such that, for any system of multiplicities $\mathbf{m}$,

$$
\varphi^{*}\left(H_{K_{q}, \mathbf{m}}\right)=H_{K_{q^{\prime}}, \mathbf{m}} .
$$

In particular, $Z_{K_{q}, \mathbf{m}} \cong Z_{K_{q^{\prime}}, \mathbf{m}}$.

Proof. Let $\mathbf{m}_{0}=\left(s-1,1^{r}\right)$. It will be enough to see that there are open neighbourhoods $V_{i} \subset S_{i-1}$ of $p_{i}$ and $V_{r+1} \subset S_{K}$ containing both $q$ and $q^{\prime}$, and isomorphisms $\varphi_{i}: V_{i} \rightarrow V_{i}$ commuting with the blowing-ups, such that $\varphi_{i}\left(p_{i}\right)=p_{i}$ and $\varphi_{r+1}(q)=q^{\prime}$. This is equivalent to prove that there are isomorphic unibranched germs of curve $\xi$ and $\xi^{\prime}$ going sharply through $\left(K_{q}, \mathbf{m}_{0}\right)$ and $\left(K_{q^{\prime}}, \mathbf{m}_{0}\right)$ respectively, because then there is a neighbourhood $V$ of $p_{1}$ where both $\xi$ and $\xi^{\prime}$ have a representative and an automorphism $\varphi$ of $V$ sending one to the other and which therefore lifts to the desired $\varphi_{i}$. Let $\xi: f=0$, respectively $\zeta: g=0$, be arbitrary unibranched germs of curve going sharply through $\left(K_{q}, \mathbf{m}_{0}\right)$, respectively $\left(K_{q^{\prime}}, \mathbf{m}_{0}\right)$. They are equisingular with a single characteristic exponent $s /(s-1)$ and their intersection 
multiplicity at $p$ is $\nu \geq(s-1)^{2}+(s-1)(s-2)-1$, so there is an automorphism of the completion

$$
\psi^{*}: \hat{\varnothing} \rightarrow \hat{\varnothing}
$$

with $\psi^{*}(f)=g$ (cf. [36, II]). Let $x, y \in \varnothing$ be a system of parameters; we have $\hat{\emptyset} \cong k[[x, y]]$, and $\psi^{*}$ can be described by

$$
\begin{aligned}
\psi^{*}(x) & =\sum_{i, j>0} a_{i j} x^{i} y^{j}, \\
\psi^{*}(y) & =\sum_{i, j>0} b_{i j} x^{i} y^{j} .
\end{aligned}
$$

Choose $e \in \mathbb{Z}_{>1}$ such that $\mathfrak{m}^{e} \subset H_{K_{q^{\prime}}, \mathbf{m}_{0}}$. Then

$$
\begin{aligned}
\varphi^{*}(x) & =\sum_{i+j \leq e} a_{i j} x^{i} y^{j}, \\
\varphi^{*}(y) & =\sum_{i+j \leq e} b_{i j} x^{i} y^{j}
\end{aligned}
$$

defines an automorphism of $\varnothing$ such that $\varphi^{*}(f)=0$ is the equation of an irreducible germ $\xi^{\prime}$ going sharply through $\left(K_{q^{\prime}}, \mathbf{m}_{0}\right)$. Indeed, $f \in \mathfrak{m}=(x, y)$ so $\varphi^{*}(f)-\psi^{*}(f) \in$ $\mathfrak{m}^{e} \subset H_{H_{q^{\prime}}, \mathbf{m}_{0}}$ and we know also that $\psi^{*}(f) \in H_{H_{q^{\prime}}, \mathbf{m}_{0}}$. Therefore, $\varphi^{*}(f)=0$ is the equation of a germ $\xi^{\prime}$ going through $\left(K_{q^{\prime}}, \mathbf{m}_{0}\right)$. As $\varphi^{*}$ is an automorphism, $\xi^{\prime}$ is irreducible and goes sharply through $\left(K_{q^{\prime}}, \mathbf{m}_{0}\right)$.

\section{SPeCializing Unibranched Cluster SCHEMES}

The subscheme of the Hilbert scheme which parametrizes cluster schemes of a given type, and its relation to the corresponding variety of clusters, has been studied by many authors ([29, [30, [31], [15], among others). However, they usually assume that the proximity relations between points of the clusters remain constant. Little seems to be known about the relative position of these subschemes. Evain computed in [11] and [12] several collisions of points, including all cases with 3 points; this is equivalent to the determination of the closure of the corresponding subschemes of the Hilbert scheme, that is, the specializations of cluster schemes when new proximity relations arise. In this section we show some flat families of unibranched cluster schemes in which proximities vary; we introduce them in order to prove Theorems 4.8 and 4.12 but they can also have some interest on their own.

Let $x, y$ be local parameters for $\varnothing$. The inclusion $\varnothing \subset \hat{\varnothing} \cong k[[x, y]]$ allows us to write any $f \in \varnothing$ as a formal power series

$$
f=\sum_{i, j \geq 0} a_{i j} x^{i} y^{j}
$$

in a unique way. Thus any polynomial $\Pi\left(X_{i j}\right) \in R=k\left[X_{i j}\right]_{i, j \geq 0}$ determines a function $\varnothing \rightarrow k$, by evaluating at $a_{i j}: \Pi(f)=\Pi\left(a_{i j}\right)_{i, j \geq 0}$, and to every $f \in \varnothing$ corresponds a maximal ideal

$$
\mathfrak{m}_{f}=\{\Pi \in R \mid \Pi(f)=0\} \subset R
$$

with quotient field equal to $k$. So we have a mapping $\varnothing \rightarrow \operatorname{MaxSpec} R \subset \operatorname{Spec} R$, and it is easy to see that it is injective. For any variety $Y$ we have therefore $\varnothing \times Y \hookrightarrow$ Spec $R \times Y$, and we shall take the Zariski topology on $\varnothing \times Y$ induced by 
the one on Spec $R \times Y$. It is easy to see that this Zariski topology does not depend on the local parameters chosen for $\varnothing$. We shall prove in 3.2 below that for any system of multiplicities $\mathbf{m}$ the set

$$
H_{\mathbf{m}}:=\left\{(f, K) \in \varnothing \times Y_{r-1} \mid f \in H_{K, \mathbf{m}}\right\} .
$$

is Zariski-closed in $\varnothing \times Y_{r-1}$.

Fix for the rest of the section a system of multiplicities $\mathbf{m}=\left(m_{1}, m_{2}, \ldots, m_{r}\right)$ which we shall apply to any cluster of $r$ points; write $\breve{\mathbf{m}}=\left(m_{1}, m_{2}, \ldots, m_{r-1}\right)$. For a cluster $K=\left(p_{1}, p_{2}, \ldots, p_{r}\right)$, we write also $\breve{K}=\left(p_{1}, p_{2}, \ldots, p_{r-1}\right)$.

Lemma 3.1. For every $K_{0} \in Y_{r-1} \subset X_{r-1}$ there are an open neighbourhood $V \subset$ $X_{r-1}$ of $K_{0}$, an isomorphism of $k$-varieties

$$
U:=V \cap Y_{r-1} \stackrel{\lambda}{\longrightarrow} k^{r-1},
$$

two functions

$$
x, y \in \Gamma\left(U \times_{Y_{r-2}} V, \varnothing_{Y_{r-1} \times_{Y_{r-2}} X_{r-1}}\right)
$$

generating the ideal of $\Delta(U)$ and polynomials $A_{i j}^{r} \in R\left[u_{1}, u_{2}, \ldots, u_{r-1}\right]$ for $i, j \geq 0$, such that for every $K \in U$ and $f \in H_{K, \mathbf{m}}$ the formal power series

$$
\sum_{i, j \geq 0} A_{i j}^{r}(f, \lambda(K))\left(x \circ i_{\breve{K}}\right)^{i}\left(y \circ i_{\breve{K}}\right)^{j}
$$

belongs to $\emptyset_{S_{\breve{K}}, p_{r}}$ and is a local equation for the virtual transform of $f=0$ at the last point $p_{r}$ of $K$.

Note that the conditions on $x, y$ and $U$ imply that for any cluster $K \in U$ the functions $x, y, \lambda_{1} \circ \pi_{U}-\lambda_{1}(K), \lambda_{2} \circ \pi_{U}-\lambda_{2}(K), \ldots, \lambda_{r-1} \circ \pi_{U}-\lambda_{r-1}(K)$ are a system of parameters for the local ring of $(K, K)$ in $U \times_{Y_{r-2}} V$, the surface

$$
\{K\} \times_{Y_{r-2}} X_{r-1}=\{K\} \times i_{\breve{K}}\left(S_{\breve{K}}\right) \cong S_{r-1}(K)
$$

is locally defined by the equations $\lambda_{i} \circ \pi_{U}=\lambda_{i}(K)$, and $x \circ i_{\breve{K}}, y \circ i_{\breve{K}}$ are local parameters for $\varnothing_{S_{r-1}, p_{r}}$ (recall that $S_{\breve{K}}=S_{r-1}(K)$ and $i_{\breve{K}}\left(p_{r}\right)=K$ ).

Proof. We proceed by induction on $r$. For $r=1$, choose a system of parameters $x, y \in \varnothing$ and a neighbourhood $V$ of $p$ where $x$ and $y$ have regular representatives (which abusing notation we call $x, y$ also) and $V(x, y) \cap V=\{p\}$. Then the claim is clear, because $Y_{0}=\{p\}$, and the virtual transform of $f$ at $p$ is $f$ itself, so we can take $A_{i j}^{1}=X_{i j}$. Suppose now $r>1$ and apply the induction hypothesis to $(\breve{K}, \breve{\mathbf{m}})$. We obtain the existence of $\breve{V} \subset X_{r-2}, \breve{U}=\breve{V} \cap Y_{r-2} \cong k^{r-2}, \breve{\lambda}, \breve{x}, \breve{y}$, and the polynomials $A_{i j}^{r-1}$ as in the claim. By making a linear substitution in $\breve{x}, \breve{y}$ we may assume that the last point $p_{r}$ of $K_{0}$ lies in the direction of $\breve{y} \circ i_{\breve{K}_{0}}=0$. $b_{r-1}^{-1}\left(\breve{U} \times_{Y_{r-3}} \breve{V}\right)$ is open in $X_{r-1}$, and is the blowing-up of $\Delta(\breve{U})$ in $\breve{U} \times_{Y_{r-3}} \breve{V}$, which can be described as the subvariety of

$$
\breve{U} \times_{Y_{r-3}} \breve{V} \times \mathbb{P}^{1}
$$

given by the equation $u \breve{x}-v \breve{y}=0$, where $(u: v)$ are projective coordinates of $\mathbb{P}^{1}$. The exceptional divisor $Y_{r-1} \cap b_{r-1}^{-1}\left(\breve{U} \times_{Y_{r-3}} \breve{V}\right)$ has equations $\breve{x}=\breve{y}=0$. We define now $V$ to be the open subset determined by $v \neq 0$, and the isomorphism

$$
\lambda: U \longrightarrow k^{r-1}=k^{r-2} \times k
$$


as $\lambda=\left(\breve{\lambda} \circ \psi_{r-1}\right) \times(u / v)$. Let $\pi_{V}, \pi_{U}$ be the projections of $U \times_{\breve{U}} V$ on its two factors. Then it is easy to see that

$$
\begin{aligned}
& x=\breve{x} \circ \pi_{V}, \\
& y=\frac{u}{v} \circ \pi_{V}-\frac{u}{v} \circ \pi_{U}=\frac{\breve{y}}{\breve{x}} \circ \pi_{V}-\lambda_{r} \circ \pi_{U}
\end{aligned}
$$

generate the ideal of $\Delta(U)$.

Because of the induction hypothesis we know that for any $K \in U$ and $f \in H_{K, \mathbf{m}}$ the virtual transform of $f$ at $p_{r-1}(K)$ relative to $\mathbf{m}$ is

$$
\breve{f}=\sum_{i, j \geq 0} A_{i j}^{r-1}\left(f, \lambda \circ \psi_{r-1}(K)\right)\left(\breve{x} \circ i_{\breve{K}}\right)^{i}\left(\breve{y} \circ i_{\breve{K}}\right)^{j}
$$

This virtual transform must have multiplicity at least $m_{r-1}$ at $p_{r-1}(K)$, therefore

$$
A_{i j}^{r-1}\left(f, \lambda \circ \psi_{r-1}(K)\right)=0 \forall i, j, i+j<m_{r-1},
$$

and the virtual transform transform of $\breve{f}$ in $S_{r-1}(K)$ is given locally by

$$
\sum_{i+j \geq m_{r-1}} A_{i j}^{r-1}\left(f, \lambda \circ \psi_{r-1}(K)\right)\left(\breve{x} \circ i_{\breve{K}} \circ \sigma_{r-1}\right)^{i+j-m_{r-1}}\left(\frac{\breve{y}}{\breve{x}} \circ i_{\breve{K}} \circ \sigma_{r-1}\right)^{j}=0
$$

which because of the commutativity $i_{\breve{K}} \circ \sigma_{r-1}=\pi_{r-1} \circ i_{\breve{K}}$ can be written in terms of the local parameters $x \circ i_{\breve{K}}, y \circ i_{\breve{K}}$ :

$$
\begin{aligned}
& \sum_{i+j \geq m_{r-1}} A_{i j}^{r-1}\left(f, \lambda \circ \psi_{r-1}(K)\right)\left(x \circ i_{\breve{K}}\right)^{i+j-m_{r-1}}\left(y \circ i_{\breve{K}}+\lambda_{r}(K)\right)^{j} \\
= & \sum_{i, j \geq m_{r-1}} \sum_{\ell=0}^{j} A_{i j}^{r-1}\left(f, \lambda \circ \psi_{r-1}(K)\right)\left(\begin{array}{c}
j \\
n
\end{array}\right) \lambda_{r}(K)^{j-\ell}\left(x \circ i_{\breve{K}}\right)^{i+j-m_{r-1}}\left(y \circ i_{\breve{K}}\right)^{\ell} .
\end{aligned}
$$

This allows us to define the polynomials

$$
A_{k \ell}^{r}\left(f, u_{1}, \ldots, u_{r-1}\right)=\sum_{i+j=k+m_{r-1}} A_{i j}^{r-1}\left(f, u_{1}, \ldots, u_{r-2}\right)\left(\begin{array}{l}
j \\
n
\end{array}\right) u_{r-1}^{j-\ell},
$$

after which the claim is satisfied.

Proposition 3.2. $H_{\mathbf{m}}$ is Zariski-closed in $\varnothing \times Y_{r-1}$.

Proof. By induction on $r$. The case $r=1$ is clear, because then $H_{K, \mathbf{m}}=\mathfrak{m}^{m_{1}}$ is the closed subset of $\varnothing=\varnothing \times Y_{0}$ determined by the ideal $I=\left(X_{i j}\right)_{i+j<m_{1}}$. Suppose now $r>1$ and the claim is true for $H_{\breve{\mathbf{m}}} \subset \varnothing \times Y_{r-2}$. Let $K_{0} \in Y_{r-1}$ be a cluster and $U \subset Y_{r-1}$ the open neighbourhood given by Lemma 3.1. It will be enough to see that $H_{U, \mathbf{m}}:=H_{\mathbf{m}} \cap \pi_{Y_{r-1}}^{-1}(U)$ is closed in $\varnothing \times U$, because $Y_{r-1}$ can be covered by a finite number of such open neighbourhoods. Define

$$
H_{U, \mathbf{m}}^{\prime}=\left\{(f, K) \in \varnothing \times U \mid\left(f, p_{r}(K)\right) \in H_{\breve{\mathbf{m}}}\right\} .
$$

Because of the induction hypothesis and the fact that

$$
i d_{\varnothing} \times p_{r}: \varnothing \times Y_{r-1} \longrightarrow \varnothing \times Y_{r}
$$


is continuous, $H_{U, \mathbf{m}}^{\prime}$ is closed in $\varnothing \times U$. Moreover, $(f, K) \in H_{U, \mathbf{m}}$ if and only if $(f, K) \in H_{U, \mathbf{m}}^{\prime}$ and the virtual transform of $f=0$ at $p_{r}(K)$ has multiplicity at least $m_{r}$, that is, by Lemma 3.1,

$$
A_{i j}^{r}(f, \lambda(K))=0 \forall i, j, i+j<m_{r} .
$$

These equations define a closed subset $V\left(A_{i j}^{r}\right)_{i+j<m_{r}} \subset \varnothing \times U$, therefore $H_{U, \mathbf{m}}=$ $H_{U, \mathbf{m}}^{\prime} \cap V\left(A_{i j}^{r}\right)_{i+j<m_{r}}$ is closed in $\varnothing \times U$.

From this we obtain a number of corollaries. Let $N$ be a positive integer such that $\operatorname{dim}\left(\varnothing / H_{K, \mathbf{m}}\right) \leq N$ for all $K \in Y_{r-1}$ (for example, $\left.N=\sum_{i=1}^{r} m_{i}\left(m_{i}+1\right) / 2\right)$. Then $H_{K, \mathbf{m}} \supset \mathfrak{m}^{N}$ for all $K$, and we can define

$$
\bar{H}_{\mathbf{m}}:=\left\{(\bar{f}, K) \in \frac{\varnothing}{\mathfrak{m}^{N}} \times Y_{r-1} \mid f \in H_{K, \mathbf{m}}\right\} .
$$

Corollary 3.3. $\bar{H}_{\mathbf{m}}$ is Zariski-closed in $\left(\varnothing / \mathfrak{m}^{N}\right) \times Y_{r-1}$.

Proof. Let $K_{0} \in Y_{r-1}$ be a cluster and $U \subset Y_{r-1}$ the open neighbourhood given by Lemma 3.1. As in Lemma 3.2, it will be enough to see that $\bar{H}_{U, \mathbf{m}}:=\bar{H}_{\mathbf{m}} \cap \pi_{Y_{r-1}}^{-1}(U)$ is closed in $\left(\varnothing / \mathfrak{m}^{N}\right) \times U$. We also know from the proof of Lemma 3.2 that $H_{U, \mathbf{m}}$ is defined by a finite number of polynomials $A_{i j}^{k}, k=1, \ldots, r$. Now for $\alpha+\beta \geq N$, $x^{\alpha} y^{\beta} \in H_{K, \mathbf{m}}$ for all $K$, so the polynomial $A_{i j}^{k}\left(x^{\alpha} y^{\beta}, u_{1}, \ldots, u_{k-1}\right) \in k\left[u_{1}, \ldots, u_{k-1}\right]$ must be identically zero. This implies in fact that

$$
A_{i j}^{k} \in k\left[X_{\alpha \beta}\right]_{\alpha+\beta<N}\left[u_{1}, u_{2}, \ldots, u_{k-1}\right] \subset R\left[u_{1}, u_{2}, \ldots, u_{k-1}\right] .
$$

Now $k\left[X_{\alpha \beta}\right]_{\alpha+\beta<N}$ is the affine coordinate ring of $\varnothing / \mathfrak{m}^{N}$, which is a $k$-vector space admitting the basis $\left\{\bar{x}^{\alpha} \bar{y}^{\beta}\right\}_{\alpha+\beta<N}$. Therefore, the $A_{i j}^{k}$ define a Zariski-closed subset in $\left(\varnothing / \mathfrak{m}^{N}\right) \times U$, and it is immediate to see that this is in fact $\bar{H}_{U, \mathbf{m}}$.

Corollary 3.4. The function

$$
\begin{aligned}
Y_{r-1} & \longrightarrow \mathbb{Z}_{\geq 0} \\
K & \longmapsto \operatorname{dim} \frac{\varnothing}{H_{K, \mathbf{m}}}=\text { length } Z_{K, \mathbf{m}}
\end{aligned}
$$

is lower semicontinuous.

Proof. Choose $N$ such that $\operatorname{dim}\left(\varnothing / H_{K, \mathbf{m}}\right) \leq N$ for all $K \in Y_{r-1}$. Then

$$
\operatorname{dim} \frac{\varnothing}{H_{K, \mathbf{m}}}=\operatorname{dim} \frac{\varnothing / \mathfrak{m}^{N}}{H_{K, \mathbf{m}} / \mathfrak{m}^{N}}=\frac{N(N+1)}{2}-\operatorname{dim}\left(\bar{H}_{\mathbf{m}} \times_{Y_{r-1}}\{K\}\right)
$$

and the claim follows because the dimension of the fibers of the (nonflat) family $\bar{H}_{\mathbf{m}} \rightarrow Y_{r-1}$ is upper semicontinuous.

Note that in general there is no ideal sheaf $\mathcal{I}$ on $S \times Y_{r-1}$ with $\mathcal{I} \otimes k(K)=H_{K, \mathbf{m}}$ for all $K$; in other words, all $Z_{K, \mathbf{m}}$ do not form a family. Indeed, the length of the members of a family of zero-dimensional schemes is upper semicontinuous, against Corollary 3.4. As the simple example $\mathbf{m}=(2,2,2)$ shows, this length is not always constant, and in fact the systems of multiplicities for which it is constant are quite exceptional. However, we shall see that restricting the set of clusters under consideration so that the length of the corresponding schemes remains constant, it is possible to construct flat families of cluster schemes. 
Given a subvariety $W \subset Y_{r-1}$, let $W_{\mathbf{m}} \subset W$ be the open subset in which $\operatorname{dim}\left(\varnothing / H_{K, \mathbf{m}}\right)$ is maximal, and denote this dimension by $N=N(\mathbf{m}, W)$. The restriction

$$
\left.\bar{H}_{\mathbf{m}}\right|_{W_{\mathbf{m}}} \subset \frac{\varnothing}{\mathfrak{m}^{N}} \times W_{\mathbf{m}}
$$

is thus a family of vector subspaces of $\varnothing / \mathfrak{m}^{N}$ of codimension $N$, so it defines a morphism $W_{\mathbf{m}} \rightarrow \mathbb{G}$ to the Grassmannian $\mathbb{G}=\mathbb{G}_{N}\left(\varnothing / \mathfrak{m}^{N}\right)$. In $\mathbb{G}$, the codimension $N$ vector spaces which are ideals of $\left(\varnothing / \mathfrak{m}^{N}\right)$ constitute a closed subscheme, which is identified to the Hilbert scheme $\operatorname{Hilb}_{p}^{N} S$ of length $N$ subschemes of $S$ supported at $p$ (cf. [13], 25]). As $H_{K, \mathbf{m}}$ is by definition an ideal for every $K$, we finally obtain a morphism

$$
\begin{gathered}
W_{\mathbf{m}} \stackrel{\varphi_{\mathbf{m}}}{\longrightarrow} \operatorname{Hilb}^{N} S \\
K \longmapsto Z_{K, \mathbf{m}}
\end{gathered}
$$

That is, the schemes $Z_{K, \mathbf{m}}$ form a flat family with parameter space $W_{\mathbf{m}}$. In the particular case that $W=C$ is a smooth curve, the morphism $\varphi_{\mathbf{m}}$ can be extended to all of $C$, by [22, III, 9.8], but then it is not true that $\varphi_{\mathbf{m}}(K)=Z_{K, \mathbf{m}}$ for those $K \in C \backslash C_{\mathbf{m}}$. In fact, $\varphi_{\mathbf{m}}(K)$ depends not only on $K$ and $\mathbf{m}$ but also on $C$. Nevertheless, we have the following

Corollary 3.5. If $C \subset Y_{r-1}$ is a smooth curve and $K \in C$, then $Z_{K, \mathbf{m}} \subseteq \varphi_{\mathbf{m}}(K)$. Proof. The morphism $\varphi_{\mathbf{m}}: C \rightarrow \operatorname{Hilb}_{p}^{N} S \subset \mathbb{G}$ defines a closed subset $\Phi_{\mathbf{m}} \subset$ $\left(\varnothing / \mathfrak{m}^{N}\right) \times C$, and we have to see that $\left.\Phi_{\mathbf{m}} \subset \bar{H}_{\mathbf{m}}\right|_{C}$. As $\bar{H}_{\mathbf{m}}$ is closed and

$$
\left.\Phi_{\mathbf{m}}\right|_{C_{\mathbf{m}}}=\left.\bar{H}_{\mathbf{m}}\right|_{C_{\mathbf{m}}} \subset \frac{\varnothing}{\mathfrak{m}^{N}} \times C_{\mathbf{m}}
$$

it will be enough to see that $\Phi_{\mathbf{m}}$ is the closure of $\left.\Phi_{\mathbf{m}}\right|_{C_{\mathbf{m}}}$.

Take $\left(\bar{f}, K_{0}\right) \in \Phi_{\mathbf{m}}$, with $K_{0} \in C \backslash C_{\mathbf{m}}$. The family determined by $\varphi_{\mathbf{m}}$ corresponds to an ideal sheaf $\mathcal{I} \subset\left(\varnothing / \mathfrak{m}^{N}\right) \otimes \varnothing_{C}$. By definition, $\bar{f} \in \mathcal{I} \otimes \varnothing_{C} k\left(K_{0}\right)$, so there is an open neighbourhood $U$ of $K_{0}$ in $C$ and a section

$$
\bar{f}_{U} \in \Gamma\left(\operatorname{Spec} \frac{\varnothing}{\mathfrak{m}^{N}} \times U, \mathcal{I}\right)
$$

with $\bar{f}=\bar{f}_{U}\left(K_{0}\right)$. As $C_{\mathbf{m}} \subset C$ is open, we may assume $U \backslash\left\{K_{0}\right\} \subset C_{\mathbf{m}}$, therefore $\forall K \in U \backslash\left\{K_{0}\right\}, \bar{f}_{U}(K) \in \mathcal{I} \otimes k(K)=\bar{H}_{K, \mathbf{m}}$. So we have a morphism

$$
\begin{aligned}
& U \longrightarrow \Phi_{\mathbf{m}} \\
& K \mapsto\left(\bar{f}_{U}(K), K\right)
\end{aligned}
$$

whose image contains $\left(\bar{f}, K_{0}\right)$ and has every other point in $\left.\Phi_{\mathbf{m}}\right|_{C_{\mathbf{m}}}$, therefore $\left(\bar{f}, K_{0}\right)$ lies in the closure of $\left.\Phi_{\mathbf{m}}\right|_{C_{\mathbf{m}}}$. As this holds for every $\left(\bar{f}, K_{0}\right) \in \Phi_{\mathbf{m}}$ and $\Phi_{\mathbf{m}}$ is closed, the claim is proved.

Next lemma shows an example of a flat family of cluster schemes which will be useful later on.

Lemma 3.6. Let $(K, \mathbf{m})$ be a consistent weighted cluster of $r$ points and $E=E_{r} \subset$ $S_{K}$ the exceptional divisor of blowing up the last point. Consider the system of multiplicities $\mathbf{m}_{1}=\left(m_{1}, m_{2}, \ldots, m_{r}, 1\right)$ and for every $q \in E, K_{q}=\left(p_{1}, p_{2}, \ldots, p_{r}, q\right)$. Then the schemes $Z_{K_{q}, \mathbf{m}_{1}}$ form a flat family. 

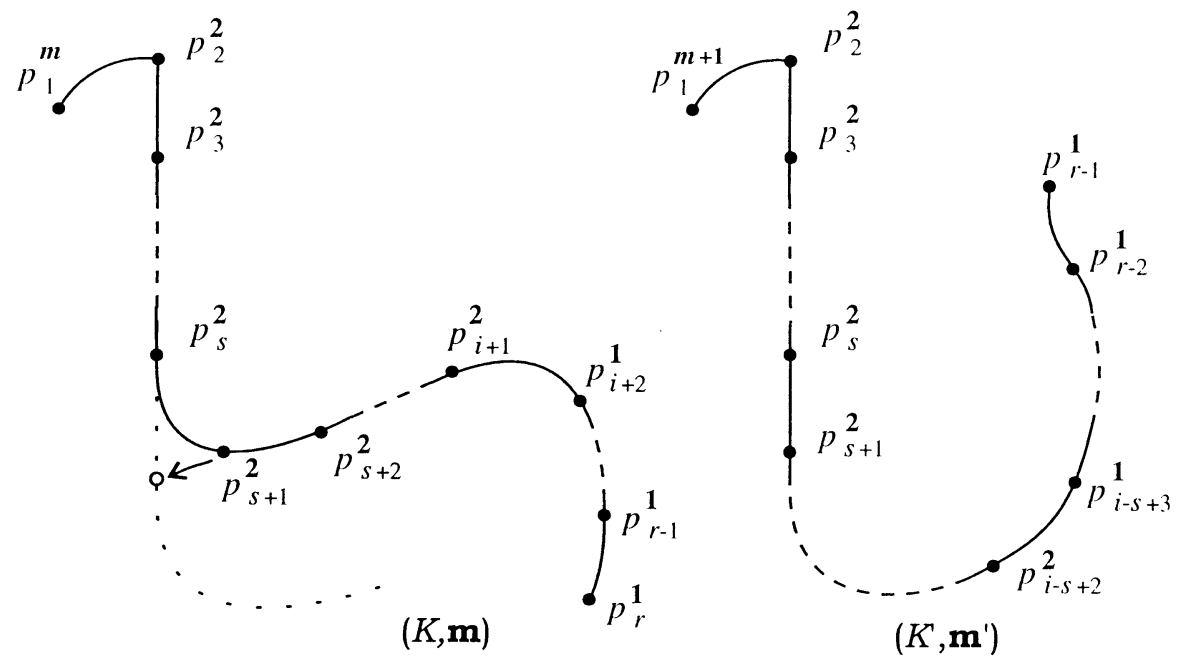

Figure 2. Enriques diagrams illustrating Proposition 3.7 By moving the free points, $Z_{K, \mathbf{m}}$ specializes to $Z_{K^{\prime}, \mathbf{m}^{\prime}}$.

Proof. It is clear that length $Z_{K_{q}, \mathbf{m}_{1}} \leq$ length $Z_{K, \mathbf{m}}+1 \forall q$. On the other hand, as $(K, \mathbf{m})$ is consistent, there are curves going sharply through $(K, \mathbf{m})$ which miss $q$ (cf. [5 4]), so length $Z_{K_{q}, \mathbf{m}_{1}}=$ length $Z_{K, \mathbf{m}}+1 \forall q$. Abusing slightly notation, we shall call $E=i_{K}(E) \subset Y_{r}$; as the length of $Z_{K_{q}, \mathbf{m}_{1}}$ does not depend on $q$, we have $E_{\mathbf{m}_{1}}=E$ and the schemes $Z_{K_{q}, \mathbf{m}_{1}}$ form a flat family.

Recall that $P_{s} \subset Y_{r-1}$ is the irreducible subvariety containing the unibranched clusters $K$ with $p_{s}(K)$ proximate to $p_{1}$, and $U_{s} \subset P_{s}$ is the open dense subset which contains all unibranched clusters based at $p_{1}$ with $p_{i}(K)$ proximate to $p_{i-1}(K)$ for all $i>1$ and to $p_{1}(K)$ if $s \geq i>1$, and no other proximity relations. Assume from now on that the system of multiplicities we are dealing with is of the form $\mathbf{m}=\left(m, 2^{i}, 1^{j}\right)$. We shall denote by $\mathcal{V}(s, m, i, j)=\overline{\varphi_{\mathbf{m}}\left(U_{s}\right)}$ the closure of the subscheme of the Hilbert scheme that parametrizes the unibranched cluster schemes $Z_{K,\left(m, 2^{i}, 1^{j}\right)}$ with $K \in U_{s}$. Note that this does not depend on $r$, as far as $r \geq i+j+1$. Note also that $U_{s} \subset\left(P_{s}\right)_{\mathbf{m}}$ because $U_{s}$ is open and dense in $P_{s}$ and length $Z_{K, \mathbf{m}}$ is constant on $U_{s}$. Therefore, $\varphi_{\mathbf{m}}\left(\left(P_{s}\right)_{\mathbf{m}}\right) \subset \overline{\varphi_{\mathbf{m}}\left(U_{s}\right)}$.

Proposition 3.7. Suppose that $\mathbf{m}=\left(m, 2^{i}, 1^{j}\right)$ is consistent in $U_{s}$ and $\left(P_{s}\right)_{\mathbf{m}}$ does not contain $U_{s+1}$. Then

1. $m=2 s-2$ and $i \geq s$,

2. if $i+j \geq s^{2}-3 s+1$, then $\mathcal{V}(s, m, i, j)$ contains $\mathcal{V}(s+1, m+1, i-s+1, j+s-2)$,

3. $\left(m+1,2^{i-s+1}, 1^{j+s-2}\right)$ is consistent in $U_{s+1}$ if and only if $i \leq 2 s-2$. If it is not, then it is equivalent to $\left(m+2,2^{i-2 s+1}, 1^{j+2 s-2}\right)$.

Proof. 1. $\mathbf{m}$ is consistent in $U_{s}$, but not consistent in $U_{s+1}$, otherwise $U_{s+1} \subset$ $\left(P_{s}\right)_{\mathbf{m}}$. Therefore, the proximity inequality at $p_{1}$ must be satisfied for $K \in U_{s}$ but not for $K \in U_{s+1}$. This means that

$$
\begin{cases}2 s>m \geq 2 s-2 & \text { if } i \geq s \\ s+i>m \geq s+i-1 & \text { if } i<s\end{cases}
$$


Therefore, if $i<s$ we must have $m=s+i-1$, and for any cluster $K \in U_{s+1}$ we have $\delta(\mathbf{m})=\left(m+1,1^{2 i+j-s}\right)$. So

$$
\begin{aligned}
& \text { length } Z_{K, \mathbf{m}}=\text { length } Z_{K, \delta(\mathbf{m})} \\
& \quad=\frac{(m+1)(m+2)}{2}+2 i+j-s=\frac{m(m+1)}{2}+3 i+j
\end{aligned}
$$

for all $K \in U_{s+1}$, so $U_{s+1} \subset\left(P_{s}\right)_{\mathbf{m}}$ against the hypotheses. We conclude that $i \geq s$, and it only remains to be seen that the case $m=2 s-1$ is not possible. But in this case $\delta(\mathbf{m})=\left(m+1,2^{i-s}, 1^{j+s}\right)$ in $U_{s+1}$, so again

$$
\begin{aligned}
& \text { length } Z_{K, \mathbf{m}}=\text { length } Z_{K, \delta(\mathbf{m})} \\
& \quad=\frac{(m+1)(m+2)}{2}+3(i-s)+j+s=\frac{m(m+1)}{2}+3 i+j
\end{aligned}
$$

for all $K \in U_{s+1}$ and $U_{s+1} \subset\left(P_{s}\right)_{\mathbf{m}}$.

2. We are given two systems of multiplicities

$$
\begin{aligned}
\mathbf{m} & =\left(2 s-2,2^{i}, 1^{j}\right), \\
\mathbf{m}^{\prime} & =\left(2 s-1,2^{i-s+1}, 1^{j+s-2}\right)
\end{aligned}
$$

with $i \geq s$ and $i+j+1 \geq(s-1)(s-2)$. We have to see that for any cluster $K_{0} \in U_{s+1}$ there is a deformation of $Z_{K_{0}, \mathbf{m}^{\prime}}$ whose general member is of the form $Z_{K, \mathbf{m}}$ with $K \in U_{s}$. For that, let $C$ be a smooth irreducible curve $K_{0} \in C \subset P_{s}$ (recall that $P_{s}$ is smooth) with $C \cap U_{s} \neq \emptyset$ and consider the morphism

$$
C \stackrel{\varphi_{\mathrm{m}}}{\longrightarrow} \operatorname{Hilb}^{N} S
$$

Consider also an auxiliary system of multiplicities and the associated morphism

$$
\begin{aligned}
\mathbf{m}_{+}= & \left(2 s-1,2^{i-s+1}, 1^{j+s-1}\right), \\
& C \stackrel{\varphi_{\mathbf{m}_{+}}}{\longrightarrow} \operatorname{Hilb}^{N+1} S .
\end{aligned}
$$

For $K \in C \cap U_{s}, Z_{K, \mathbf{m}} \varsubsetneqq Z_{K, \mathbf{m}_{+}}$. Therefore, $\varphi_{\mathbf{m}}(K) \varsubsetneqq \varphi_{\mathbf{m}_{+}}(K)$ for all $K \in C$. Moreover, part 1 of the proposition shows that $K_{0} \in C_{\mathbf{m}_{+}}$, so $\varphi_{\mathbf{m}_{+}}\left(K_{0}\right)=$ $Z_{K_{0}, \mathbf{m}_{+}}$. On the other hand, $\left(K_{0}, \mathbf{m}\right)$ does not satisfy the proximity inequality at $p_{1}$, and unloading multiplicity at this point gives

$$
\mathbf{m}_{-}=\left(2 s-1,2^{i-s}, 1^{j+s}\right) .
$$

Note that $\mathbf{m}_{-}$may be nonconsistent in $U_{s+1}$, but still $Z_{K_{0}, \mathbf{m}}=Z_{K_{0}, \mathbf{m}_{-}}$and length $Z_{K_{0}, \mathbf{m}}<$ length $\varphi_{\mathbf{m}}\left(K_{0}\right)$. All together, we have

$$
Z_{K_{0}, \mathbf{m}_{-}}=Z_{K_{0}, \mathbf{m}} \varsubsetneqq \varphi_{\mathbf{m}}\left(K_{0}\right) \varsubsetneqq \varphi_{\mathbf{m}_{+}}\left(K_{0}\right)=Z_{K_{0}, \mathbf{m}_{+}}
$$

and we are in the conditions of Lemma 2.1 Therefore, there is a point $q$ in the exceptional divisor $E=E_{r}$ of blowing up $p_{r}$ such that $\varphi_{\mathbf{m}}\left(K_{0}\right)=Z_{K_{q}, \mathbf{m}_{0}}$, with

$$
\mathbf{m}_{0}=\left(2 s-1,2^{i-s}, 1^{j+s+1}\right) .
$$

Let $q_{0} \in E$ be the unique point proximate to $p_{r-1}$. If $q=q_{0}$, then unloading gives $Z_{K_{q}, \mathbf{m}_{0}}=Z_{K_{0}, \mathbf{m}^{\prime}}$ so $\varphi_{\mathbf{m}}$ is the family we are looking for. If $q$ is not proximate to $p_{r-1}$, then we only know that $Z_{K_{q}, \mathbf{m}_{0}} \in \mathcal{V}(s, m, i, j)$. As $i+j+$ $1 \geq(s-1)(s-2)$, though, by Lemma 2.4 we can say that for any free point 
$q \in E, Z_{K_{q}, \mathbf{m}_{0}} \in \mathcal{V}(s, m, i, j)$. As the free points are dense in $E$, this implies $\varphi_{\mathbf{m}_{0}}(E) \subset \mathcal{V}(s, m, i, j)$. Now it is enough to see that $q_{0} \in E_{\mathbf{m}_{0}}$, because then $Z_{K_{0}, \mathbf{m}^{\prime}}=Z_{K_{q_{0}}, \mathbf{m}_{0}}=\varphi_{\mathbf{m}_{0}}\left(q_{0}\right) \in \mathcal{V}(s, m, i, j)$. If $\left(K_{q}, \mathbf{m}_{0}\right)$ is consistent for $q \in$ $E$ free, then $q_{0} \in E_{\mathbf{m}_{0}}$ because of Lemma 3.6] if it is not, then the equivalent consistent system obtained by unloading is $\delta_{K_{q}}\left(\mathbf{m}_{0}\right)=\left(m+2,2^{i-2 s}, 1^{j+2 s+1}\right)$, whereas for the cluster $K_{q_{0}}$ the equivalent consistent system is $\delta_{K_{q_{0}}}\left(\mathbf{m}_{0}\right)=$ $\left(m+2,2^{i-2 s+1}, 1^{j+2 s-2}\right)$. This implies that length $Z_{K_{q_{0}}, \mathbf{m}_{0}}=N\left(\mathbf{m}_{0}, E\right)$, so again $q_{0} \in E_{\mathbf{m}_{0}}$.

3. Follows from an easy unloading calculation.

Corollary 3.8. Suppose that $\mathbf{m}=\left(m, 2^{i}, 1^{j}\right)$ is consistent in $U_{s}$ and $\left(P_{s}\right)_{\mathbf{m}}$ does not contain $U_{s+1}$, and suppose that $j \geq s^{2}-5 s+2$. For every $k \in \mathbb{Z}_{\geq 0}$ we define

$$
\begin{aligned}
m_{k} & =m+2 k, \\
i_{k} & =i-k(k+2 s-2), \\
j_{k} & =j+k(k+2 s-3) .
\end{aligned}
$$

If either $k=0$ or $i_{k-1}>2(s+k-1)-2$, then $\mathcal{V}\left(s+k, m_{k}, i_{k}, j_{k}\right) \subset \mathcal{V}(s, m, i, j)$.

Proof. We shall proceed by induction on $k$. For $k=0$, there is nothing to prove. For $k>0$, we have either $k-1=0$ or

$$
i_{k-2}>i_{k-1}>2(s+k-1)-2>2(s+k-2)-2
$$

so we can apply the induction hypothesis and

$$
\mathcal{V}\left(s+k-1, m_{k-1}, i_{k-1}, j_{k-1}\right) \subset \mathcal{V}(s, m, i, j) .
$$

On the other hand, a straightforward computation shows that $j_{k-1} \geq(s+k-1)^{2}-$ $5(s+k-1)+2$, therefore $i_{k-1}+j_{k-1} \geq(s+k-1)^{2}-3(s+k-1)+1$. Under the conditions, Proposition 3.7 tells us that

$$
\mathcal{V}\left(s+k, m_{k}, i_{k}, j_{k}\right) \subset \mathcal{V}\left(s+k-1, m_{k-1}, i_{k-1}, j_{k-1}\right),
$$

thus finishing the proof.

\section{Degree of Singular Plane Curves}

In this section we work on a projective irreducible smooth surface $S$, mainly $S=$ $\mathbb{P}^{2}$. We are interested in linear systems of curves which contain a zero-dimensional scheme composed of unibranched cluster schemes. These linear systems can be specialized to linear systems of curves through a unibranched cluster (supported at a single point) using the technique developed in 33. We shall not repeat the whole treatment here, but only show the parameter space (a variety of clusters) to which we apply the semicontinuity theorem.

For convenience, we fix a point $p$ in $S$. Given a sequence of integers $\mathbf{r}=$ $\left(r_{1}, r_{2}, \ldots, r_{\rho}\right)$ with $r_{1}<r_{2}<\ldots<r_{\rho}$ we define varieties $X_{i}(\mathbf{r})$ and $Y_{i}(\mathbf{r})$ in an analogous way to $X_{i}$ and $Y_{i}$. Let $Y_{-1}(\mathbf{r})=\operatorname{Spec} k, X_{0}(\mathbf{r})=S, Y_{0}(\mathbf{r})=\{p\}$, and for $i>0$ let

$$
X_{i}(\mathbf{r}) \stackrel{b_{i}}{\longrightarrow} Y_{i-1}(\mathbf{r}) \times_{Y_{i-2}(\mathbf{r})} X_{i-1}(\mathbf{r})
$$

be the blowing-up along $\Delta\left(Y_{i-1}(\mathbf{r})\right)$,

$$
Y_{i}(\mathbf{r})= \begin{cases}\text { exc. divisor of the blowing-up } & \text { if } i \notin\left\{r_{1}, r_{2}, \ldots, r_{\rho}\right\}, \\ X_{i}(\mathbf{r}) & \text { if } i \in\left\{r_{1}, r_{2}, \ldots, r_{\rho}\right\}\end{cases}
$$


and $\psi_{i}(\mathbf{r})=\pi_{Y_{i-1}(\mathbf{r})} \circ b_{i}: X_{i}(\mathbf{r}) \rightarrow Y_{i-1}(\mathbf{r})$ is smooth of relative dimension 2 . We write also $X(\mathbf{r})=X_{r_{\rho}}(\mathbf{r})$ and $Y(\mathbf{r})=Y_{r_{\rho}-1}(\mathbf{r})$. All these varieties are projective, irreducible and smooth; $Y(\mathbf{r})$ can be identified with the set of all clusters of $r_{\rho}$ points of $S$, such that $p_{1}=p$ and $p_{i+1}$ is proximate to $p_{i}$ for all $i \notin \mathbf{r}$. A general cluster $K \in Y(\mathbf{r})$ is the union of $\rho$ unibranched clusters of $r_{1}, r_{2}-r_{1}, \ldots$, and $r_{\rho}-r_{\rho-1}$ points.

Lemma 4.1. For any sequence of integers $\mathbf{r}=\left(r_{1}, r_{2}, \ldots, r_{\rho}\right), X_{i}$ and $Y_{i}$ are closed subvarieties of $X_{i}(\mathbf{r})$ and $Y_{i}(\mathbf{r})$ respectively, and the morphism $\psi_{i}: X_{i} \rightarrow Y_{i-1}$ of section 2 is the restriction of $\psi_{i}(\mathbf{r}): X_{i}(\mathbf{r}) \rightarrow Y_{i-1}(\mathbf{r})$ for all $i$.

Proof. By induction on $i$. The cases $i \leq 1$ are clear, so assume $i>1$ and the claim to be true for $X_{i-1}$ and $X_{i-2}$. This means that $X_{i-1} \subset X_{i-1}(\mathbf{r}), Y_{i-1} \subset Y_{i-1}(\mathbf{r})$ are closed subvarieties and the morphism $\psi_{i-1}: X_{i-1} \rightarrow Y_{i-2}$ is the restriction of $\psi_{i-1}(\mathbf{r}): X_{i-1}(\mathbf{r}) \rightarrow Y_{i-2}(\mathbf{r})$. So we have also a closed subvariety

$$
Y_{i-1} \times_{Y_{i-2}} X_{i-1} \subset Y_{i-1}(\mathbf{r}) \times_{Y_{i-2}(\mathbf{r})} X_{i-1}(\mathbf{r})
$$

and

$$
\Delta\left(Y_{i-1}(\mathbf{r})\right) \cap Y_{i-1} \times_{Y_{i-2}} X_{i-1}=\Delta\left(Y_{i-1}\right)
$$

so by the definitions, $X_{i} \subset X_{i-1}(\mathbf{r})$ is the strict transform of $Y_{i-1} \times_{Y_{i-2}} X_{i-1}$ under the blowing-up $b_{i}$ (cf. [22, II.7.15]), $Y_{i}$ is a subvariety of the exceptional divisor, hence of $Y_{i-1}(\mathbf{r})$, and $\psi_{i}: X_{i} \rightarrow Y_{i-1}$ is the restriction of $\psi_{i}: X_{i}(\mathbf{r}) \rightarrow Y_{i-1}(\mathbf{r})$.

Given a cluster $K \in Y(\mathbf{r})$, a system of multiplicities $\mathbf{m}$ and a divisor $D$, we shall denote by $\mathrm{E}_{D}(K, \mathbf{m})$ the linear system of effective divisors linearly equivalent to $D$ which go through $(K, \mathbf{m})$. As seen in 33 the function

$$
\begin{aligned}
Y(\mathbf{r}) & \longrightarrow \mathbb{Z}_{\geq-1} \\
K & \longmapsto \ell_{D}(K, \mathbf{m}):=\operatorname{dim} \mathrm{E}_{D}(K, \mathbf{m})
\end{aligned}
$$

is upper semicontinuous. We consider the dimension of the empty linear system to be -1 . Because of Lemma 4.1, we can bound $\ell_{D}(K, \mathbf{m})$ with $K$ general in $Y(\mathbf{r})$ by $\ell_{D}(K, \mathbf{m})$ with $K$ in $Y_{r_{\rho}-1}$. For any subset $W \subset Y(\mathbf{r})$ we shall also write $\ell_{D}(W, \mathbf{m})=\inf \left\{\ell_{D}(K, \mathbf{m}) \mid K \in W\right\}$.

In the case $S=\mathbb{P}^{2}$, we can choose $D=d \cdot L$, with $L$ a line and $d \in \mathbb{Z}_{>0}$. We shall then denote $\mathrm{七}_{d}(K, \mathbf{m})=\mathrm{七}_{D}(K, \mathbf{m})$ the linear system of curves of degree $d$ going through $(K, \mathbf{m})$ and $\ell_{d}(K, \mathbf{m})=\ell_{D}(K, \mathbf{m})$ its dimension. We say that a zero-dimensional scheme $Z$ has level $d$ when length $Z=(d+1)(d+2) / 2$. For a level $d$ scheme in the plane $\mathbb{P}^{2}$ it is equivalent to have maximal rank or to have maximal rank in degree $d$. Furthermore, an arbitrary zero-dimensional subscheme $Z \subset \mathbb{P}^{2}$ has maximal rank if and only if there exist maximal rank schemes $Z_{d}$ and $Z_{d+1}$ of level $d$ and $d+1$ respectively such that $Z_{d} \subset Z \subset Z_{d+1}$ (see [23, 2.2.2]).

Lemma 4.2. For every cluster scheme $Z_{K, \mathbf{m}}$ where $K \in U_{s}$ and $\mathbf{m}=\left(m, 2^{i}, 1^{j}\right)$ is consistent in $U_{s}$ there exist systems of multiplicities $\mathbf{m}_{-}=\left(m, 2^{i_{-}}, 1^{j_{-}}\right)$and $\mathbf{m}_{+}=\left(m, 2^{i_{+}}, 1^{j_{+}}\right)$consistent in $U_{s}$ such that $Z_{K, \mathbf{m}_{-}} \subset Z_{K, \mathbf{m}} \subset Z_{K, \mathbf{m}_{+}}, Z_{K, \mathbf{m}_{-}}$ has level $d$ and $Z_{K, \mathbf{m}_{+}}$has level $d+1$. Furthermore, $\mathbf{m}_{+}$and $\mathbf{m}_{-}$can be chosen such that $j_{+} \geq j$ and $\left(j_{-} \geq j\right.$ or $\left.i_{-}=0\right)$, the inequalities being strict if $Z_{K, \mathbf{m}}$ does not have level $d$. If $3 i+j \geq 2 m+3$, then $d>m$. 
Proof. Write

$$
\frac{m(m+1)}{2}+3 i+j=\frac{(d+1)(d+2)}{2}+\epsilon
$$

with $0 \leq \epsilon \leq d+1$. It is enough to define $\mathbf{m}_{+}=\left(m, 2^{i}, 1^{j+d+2-\epsilon}\right)$ and

$$
\mathbf{m}_{-}= \begin{cases}\left(m, 2^{i-\epsilon}, 1^{j+2 \epsilon}\right) & \text { if } i \geq \epsilon \\ \left(m, 1^{j+3 i-\epsilon}\right) & \text { if } i \leq \epsilon .\end{cases}
$$

Before we prove our maximal rank theorem we need some lemmas on level $d$ cluster schemes. Assume for a while that $\mathbf{m}=\left(m, 2^{i}, 1^{j}\right)$ and $d \in \mathbb{Z}$ are such that

$$
N(\mathbf{m})=\frac{m(m+1)}{2}+3 i+j=\frac{(d+1)(d+2)}{2} .
$$

Lemma 4.3. If $i=0$ and $s \leq m+1$, then $\ell_{d}\left(U_{s}, \mathbf{m}\right)=-1$, with $U_{s} \subset Y_{r_{\rho}-1}$ as defined in section 3

Proof. It is clear that $d \geq m-1$; we shall prove the claim by induction on $d-m$. For $d=m-1$ the result is obvious. For $d \geq m$ we have $j \geq m+1$. By semicontinuity it is enough to see that there are no plane curves of degree $d$ containing $Z_{K, \mathbf{m}}$ with $K$ general in $U_{m+2}$. Now for $K \in U_{m+2}$, unloading gives $Z_{K, \mathbf{m}}=Z_{K,\left(m+1,1^{j-m-1}\right)}$, and the result follows from the induction hypothesis.

Lemma 4.4. If $2 i \leq m, 2 i+j>m$ and $\mathbf{m}$ is consistent in $U_{s}$, then $\ell_{d}\left(U_{s}, \mathbf{m}\right)=$ -1 .

Proof. By semicontinuity it is enough to see that there are no plane curves of degree $d$ containing $Z_{K, \mathbf{m}}$ with $K$ general in $U_{m+2-i}$. But for $K \in U_{m+2-i}$, unloading gives $Z_{K, \mathbf{m}}=Z_{K,\left(m+1,1^{2 i+j-m-1}\right)}$, and the result follows from Lemma 4.3 .

Proposition 4.5. If $d \leq m+1$ and $\mathbf{m}$ is consistent in $U_{s}$, then

$$
\ell_{d}\left(U_{s}, \mathbf{m}\right)=-1 \Longleftrightarrow\left\{\begin{array}{l}
d \leq m \text { and } i=0 \\
\text { or } \\
d=m+1 \text { and } m \geq i+s-2 .
\end{array}\right.
$$

Proof. It is clear that $d \geq m-1$, and $d=m-1$ implies $i=j=0, \ell_{d}\left(U_{s}, \mathbf{m}\right)=-1$. Assume now $d=m$ or $d=m+1$. We will prove the claim by induction on $i$. If $i=0$, then consistency guarantees both $m \geq i+s-2$ and $s \leq m+1$, so the result follows from Lemma 4.3. Assume now $i \geq 1$. For $K$ general in $U_{s}$, consider the line $L$ passing through $p$ in the direction of $p_{2}$. If $s=2$, we can assume ( $K$ being general) that $p_{3}$ does not lie on $L$; for $s>2$ this is automatic. For every curve $C \in \mathrm{E}_{d}(K, \mathbf{m})$

$$
L \cdot C \geq m+2=d+1>\operatorname{deg} C,
$$

therefore $L$ is a component of $C$, so it is a fixed part of $\mathrm{E}_{d}(K, \mathbf{m})$. The residual linear system is

$$
\mathrm{Ł}_{d}(K, \mathbf{m})-L=\mathrm{E}_{d-1}\left(K, \mathbf{m}^{\prime}\right)
$$


with $\mathbf{m}^{\prime}=\left(m-1,1,2^{i-1}, 1^{j}\right)$ because of Corollary 2.3 As this is true for general $K \in U_{s}$, we have now $\ell_{d}\left(U_{s}, \mathbf{m}\right)=\ell_{d-1}\left(U_{s}, \mathbf{m}^{\prime}\right)$. But $\mathbf{m}^{\prime}$ is not consistent in $U_{s}$. The equivalent consistent system is

$$
\delta\left(\mathbf{m}^{\prime}\right)= \begin{cases}\left(m, 2^{i-s}, 1^{j+s}\right) & \text { if } m=2(s-1) \leq 2(i-1), \\ \left(m-1,2^{i-1}, 1^{j+1}\right) & \text { in any other case. }\end{cases}
$$

Note that $N\left(\delta\left(\mathbf{m}^{\prime}\right)\right)=N(\mathbf{m})-m-2$, so

1. If $d=m$, then $N\left(\delta\left(\mathbf{m}^{\prime}\right)\right)<d(d+1) / 2$ and $\mathrm{七}_{d}(K, \mathbf{m})=\mathrm{七}_{d-1}\left(K, \delta\left(\mathbf{m}^{\prime}\right)\right)+L$ is nonempty for all $K \in U_{s}$, as claimed for $d=m, i \neq 0$.

2. If $d=m+1$, then $N\left(\delta\left(\mathbf{m}^{\prime}\right)\right)=d(d+1) / 2$ so $Z_{K, \mathbf{m}}$ has level $d-1$ for all $K \in$ $U_{s}$. Now the induction hypothesis gives $\ell_{d}\left(U_{s}, \mathbf{m}\right)=\ell_{d-1}\left(U_{s}, \delta\left(\mathbf{m}^{\prime}\right)\right)=-1$ because

$$
m \geq i+s-2 \Longleftrightarrow \begin{cases}i-s=0 & \text { if } m=2(s-1) \leq 2(i-1) \\ m-1 \geq(i-1)+s-2 & \text { in any other case. }\end{cases}
$$

Corollary 4.6. If $2 i \leq m, d>m$ and $\mathbf{m}$ is consistent in $U_{s}$, then $\ell_{d}\left(U_{s}, \mathbf{m}\right)=-1$.

Proof. By Lemma 4.4 we may assume $2 i+j \leq m$. But then

$$
N(\mathbf{m})=\frac{m(m+1)}{2}+3 i+j \leq \frac{m(m+1)}{2}+m+\frac{m}{2}<\frac{(m+3)(m+4)}{2}
$$

so $d<m+2$. Moreover, we have either $i \geq s-2$, in which case $m \geq 2 i \geq i+s-2$ or $i \leq s-2$, in which case consistency of $\mathbf{m}$ in $U_{s}$ implies $m \geq i+s-2$. In both cases Proposition 4.5 concludes.

Lemma 4.7. If $i \leq m, d>m, 4(i+j) \geq m^{2}-2 m-4$ and $\mathbf{m}$ is consistent in $U_{s}$, then $\ell_{d}\left(U_{s}, \mathbf{m}\right)=-1$, except in the case $m=6, i=5, j=0, s=4$.

Proof. Consider first the case $d=m+1$. If $m \geq i+s-2$, then Proposition 4.5 says that $\ell_{d}\left(U_{s}, \mathbf{m}\right)=-1$, as wanted, so assume $m<i+s-2$. We will see that this implies $m=6, i=5, j=0, s=4$. Indeed, $d=m+1$ implies $3 i+j=2 m+3$, so $j=2 m+3-3 i$ and $2 m+3-3 i$ cannot be negative. The conditions $2 m+3-3 i \geq 0$ and $4(i+j)=8 m-8 i+12 \geq m^{2}-2 m-4$ allow only a small finite number of solutions $(m, i)$, and $(m, i)$ determine $j$. Moreover, $s$ is limited by consistency, so we only have to check a finite number of 4 -ples $(m, i, j, s)$ to see that the only one with $m<i+s-2$ is $(6,5,0,4)$.

Assume now $d>m+1$. We shall use semicontinuity applied to a family of level $d$ cluster schemes which specializes free points to be proximate to $p_{1}$. When $m$ is odd, unloading alone is enough to obtain the family; for even $m$ we will use Proposition 3.7. By Corollary 4.6 we may assume $2 i>m$; now we distinguish two cases according to the parity of $m$ :

$m$ even Write $m=2 t-2$. As $\mathbf{m}$ is consistent in $U_{s}$, and $2 i>m$, we must have $t \geq s$, so by semicontinuity it is enough to see that there are no plane curves of degree $d$ containing $Z_{K, \mathbf{m}}$ with $K$ general in $U_{t}$. It is clear that $\left(P_{t}\right)_{\mathbf{m}}$ does not contain $U_{t+1}$, and $4(i+j) \geq 4\left(t^{2}-3 t+1\right)$, therefore by Proposition 3.7

$$
\mathcal{V}(t+1, m+1, i-t+1, j+t-2) \subset \mathcal{V}(t, m, i, j) .
$$

By semicontinuity applied to the tautological flat family on $\mathcal{V}(t, m, i, j) \subset$ $\operatorname{Hilb}^{N}(S)$ then, it is enough to see that there are no plane curves of degree $d$ 
containing $Z_{K, \mathbf{m}^{\prime}}$ with $K$ general in $U_{t+1}$ and $\mathbf{m}^{\prime}=\left(m+1,2^{i-t+1}, 1^{j+t-2}\right)$. But then $2(i-t+1)=2 i-m \leq m+1$ and still $d>m+1$ so the result follows by Corollary 4.6

$m$ odd Write $m=2 t-1$. By semicontinuity it is enough to see that there are no plane curves of degree $d$ containing $Z_{K, \mathbf{m}}$ with $K$ general in $U_{t+1}$. But for $K \in U_{t+1}$, unloading gives $Z_{K, \mathbf{m}}=Z_{K,\left(m+1,2^{i-t}, 1^{j+t}\right)}$, and again $2(i-t)=2 i-m-1 \leq m+1$ and $d>m+1$ so the result follows by Corollary 4.6 .

With this knowledge of level $d$ cluster schemes we are in a position to attack the general case. Now let $\rho, s$ be positive integers, and suppose we have systems of multiplicities $\mathbf{m}_{1}, \mathbf{m}_{2}, \ldots, \mathbf{m}_{\rho}$ with

$$
\begin{aligned}
& \mathbf{m}_{1}=\left(m, 2^{i_{1}}, 1^{j_{1}}\right), \\
& \mathbf{m}_{k}=\left(2^{i_{k}}, 1^{j_{k}}\right), \quad k=2,3, \ldots, \rho .
\end{aligned}
$$

Suppose furthermore that $m \geq \min \left(s-1+\sum i_{k}, 2(s-1)\right)$. Consider a cluster scheme $Z_{1}=Z\left(K, \mathbf{m}_{1}\right)$ with $K \in U_{s}$ and $\rho-1$ unibranched cluster schemes $Z_{2}, Z_{3}, \ldots, Z_{\rho}$ supported at different points of $\mathbb{P}^{2}$, whose defining clusters $K_{2}, K_{3}, \ldots, K_{\rho}$ have no satellite points. The scheme $Z=Z_{1} \cup Z_{2} \cup \cdots \cup Z_{\rho}$ has length

$$
N=\frac{m(m+1)}{2}+3 \sum_{k=1}^{\rho} i_{k}+\sum_{k=1}^{\rho} j_{k} .
$$

Theorem 4.8. If the position of the points of the $\rho$ clusters $K_{2}, K_{3}, \ldots, K_{\rho}$ is general, $3 \sum i_{k}+\sum j_{k} \geq 2 m+3$, and $4 \sum j_{k} \geq m^{2}-4 m-6$, then $Z$ has maximal rank, except in the cases

- $m=2, \sum i_{k}=4, j_{k}=0 \forall k$,

- $m=4, \sum i_{k}=6, j_{k}=0 \forall k$,

- $m=6, \sum i_{k}=5, j_{k}=0 \forall k, s=4$.

Proof. Consider $\mathbf{r}=\left(r_{1}, r_{2}, \ldots, r_{\rho}\right)$. Let $P_{s}(\mathbf{r})$ be the closed variety of $Y(\mathbf{r})$ where $p_{2}, p_{3}, \ldots, p_{s}$ are proximate to $p$. It is easy to see that $P_{s}(\mathbf{r})$ is an irreducible variety and $P_{s}=P_{s}(\mathbf{r}) \cap Y_{r_{\rho}-1}$. Then the claim may be equivalently stated as

$$
\ell_{d}\left(P_{s}(\mathbf{r}), \mathbf{m}_{0}\right)=\max \left(-1, \frac{(d+1)(d+2)}{2}-N\right) \forall d
$$

where $\mathbf{m}_{0}=\left(m, 2^{i_{1}}, 1^{j_{1}}, 2^{i_{2}}, 1^{j_{2}}, \ldots, 2^{i_{\rho}}, 1^{j_{\rho}}\right)$. By semicontinuity it is enough to see that $\ell_{d}\left(P_{s}, \mathbf{m}_{0}\right)=\max (-1,(d+1)(d+2) / 2-N) \forall d$ and unloading gives that $\mathbf{m}=\left(m, 2^{\sum i_{k}}, 1^{\sum j_{k}}\right)$ is equivalent to $\mathbf{m}_{0}$ in $U_{s}$. Therefore,

$$
\ell_{d}\left(P_{s}(\mathbf{r}), \mathbf{m}_{0}\right) \leq \ell_{d}\left(U_{s}, \mathbf{m}_{0}\right)=\ell_{d}\left(U_{s}, \mathbf{m}\right) \forall d .
$$

It only remains to be seen that for a general cluster $K \in U_{s}, Z_{K, \mathbf{m}}$ has maximal rank. Because of Lemma 4.2 either $Z_{K, \mathbf{m}}$ has level $d$ or there are schemes $Z_{K, \mathbf{m}_{-}}$ and $Z_{K, \mathbf{m}_{+}}$of level $d$ and $d+1$ respectively, satisfying the above conditions and not among the exceptions, with $Z_{K, \mathbf{m}_{-}} \subset Z_{K, \mathbf{m}} \subset Z_{K, \mathbf{m}_{+}}$, so we can assume that $Z_{K, \mathbf{m}}$ has level $d>m$, and it is enough to see that $\ell_{d}\left(U_{s}, \mathbf{m}\right)=-1$.

Let $i=\sum i_{k}, j=\sum j_{k}$. The proof runs by induction on $i$. The case $i \leq m$ has already been settled in Lemma4.7, so suppose $i \geq m+1$. We shall distinguish two cases according to the parity of $m$. 
$m$ even Write $m=2 t-2$. As $\mathbf{m}$ is consistent in $U_{s}$ and $i \geq m+1$, we must have $t \geq s$, so by semicontinuity it is enough to see that there are no plane curves of degree $d$ containing $Z_{K, \mathbf{m}}$ with $K$ general in $U_{t}$. It is clear that $\left(P_{t}\right)_{\mathbf{m}}$ does not contain $U_{t+1}$, and $4(i+j) \geq 4\left(t^{2}-3 t+1\right)$, therefore by Proposition 3.7

$$
\mathcal{V}(t+1, m+2, i-2 t+1, j+2 t-2) \subset \mathcal{V}(t, m, i, j) .
$$

By semicontinuity applied to the tautological flat family on $\mathcal{V}(t, m, i, j) \subset$ $\operatorname{Hilb}^{N}(S)$, then it is enough to see that there are no plane curves of degree $d$ containing $Z_{K, \mathbf{m}^{\prime}}$ with $K$ general in $U_{t+1}$ and

$$
\mathbf{m}^{\prime}=\left(m^{\prime}, 2^{i^{\prime}}, 1^{j^{\prime}}\right)=\left(m+2,2^{i-2 t+1}, 1^{j+2 t-2}\right) .
$$

An easy computation shows that we are still in the numerical conditions of the claim, and $i^{\prime}<i$, so we can apply the induction hypothesis, except in the case that $d=m+2$. In this case, either we are in one of the exceptions above, or $i=m+1, j=3, \mathbf{m}^{\prime}=\left(m+2,1^{m+3}\right)$ and the claim follows from Lemma 4.3

$m$ odd Write $m=2 t-1$. By semicontinuity it is enough to see that there are no plane curves of degree $d$ containing $Z_{K, \mathbf{m}}$ with $K$ general in $U_{t+1}$. But for $K \in U_{t+1}$, unloading gives $Z_{K, \mathbf{m}}=Z_{K,\left(m+1,2^{i-t}, 1^{j+t}\right)}$, so the result follows by the induction hypothesis again.

Note that Theorem 4.8 applies in particular when we have only double points, proper or infinitely near, and they are neither 5 nor less than 4 . For the remaining cases the behaviour is also known (cf. [17]). Namely, one double point has (obviously) maximal rank and the scheme of three double points (proper or infinitely near, in general position) has maximal rank; systems which fail to have maximal rank appear in degree 2 when there are two points and in degree 4 when there are five. In both cases there is an "unexpected" curve of the form $C=2 D$, where $D$ is the straight line or the conic through the points, respectively. The cases of one point of multiplicity $m=3,4$ or 5 and $i$ double points (again, proper or infinitely near) are also covered, except for $i<2 m / 3+1$ and for the case $m=4, i=6$. All excepted cases involve less than eight points and were therefore also solved by B. Harbourne. The systems which do not have maximal rank are $(3,2)$ in degree $3,(4,2),\left(4,2^{2}\right)$ in degree $4,(5,2),\left(5,2^{2}\right)$ in degree 5 , and $\left(4,2^{6}\right)$ in degree 6 .

We shall now apply this result to find irreducible curves of low degree with tacnodes and cusps. We shall use a form of Bertini's theorems slightly different from the usual ones. Given a linear system $€$ of curves on $S$ with no fixed part, the base points of $€$ form a (usually nonunibranched) cluster $\mathrm{BP}(\mathrm{E})$.

Proposition 4.9. Let $E$ be a linear system of curves on $S$ with no fixed part. Then

1. General curves in $E$ go sharply through $\mathrm{BP}(E)$.

2. If $\mathrm{E}$ is reducible, then it is composed of the curves of a pencil.

Proof. 2 and the fact that all singularities of general curves sit at the proper base points of $\mathrm{E}$ are standard. For a proof of 1, cf. [5, 7.2]. Cf. also Zariski's remark on the theorems of Bertini in [35].

Lemma 4.10. Let $Z \subset Z^{\prime}$ be two zero-dimensional subschemes of $\mathbb{P}^{2}$ such that length $Z^{\prime}=$ length $Z+1$, and let $d$ be a positive integer such that going through $Z$ imposes independent conditions to curves of degree $d$. Then $Z$ ' imposes independent conditions to curves of degree $d+1$. 
Proof. This is an easy application of the residual exact sequence of the Horace method. There is a unique point $p \in \mathbb{P}^{2}$ where the length of the component of $Z^{\prime}$ supported at $p$ is bigger than that of $Z$. Let $L$ be a general straight line through $p$, and consider the residual exact sequence

$$
0 \longrightarrow \mathcal{I}_{Z^{\prime \prime}}(d) \longrightarrow \mathcal{I}_{Z^{\prime}}(d+1) \longrightarrow \mathcal{I}_{Z^{\prime} \cap L / L}(d+1) \longrightarrow 0 .
$$

We have to prove that

$$
H^{1}\left(\mathcal{I}_{Z^{\prime} \cap L / L}(d+1)\right)=H^{1}\left(\mathcal{I}_{Z^{\prime \prime}}(d)\right)=0 .
$$

As going through $Z$ imposes independent conditions to curves of degree $d$ and $L$ is general, length $Z \cap L \leq d+1$, therefore length $Z^{\prime} \cap L \leq d+2$ and

$$
H^{1}\left(\mathcal{I}_{Z^{\prime} \cap L / L}(d+1)\right)=0 .
$$

As by hypothesis $H^{1}\left(\mathcal{I}_{Z}(d)\right)=0$, it will be enough to see that $Z$ contains the residual scheme $Z^{\prime \prime}$. Let $I, I^{\prime}, I^{\prime \prime} \subset \varnothing_{p}$ be the ideals locally defining $Z, Z^{\prime}$ and $Z^{\prime \prime}$, and let $f \in \varnothing_{p}$ be a local equation of $L$. length $Z^{\prime}=$ length $Z+1$ implies that $I=I^{\prime}+(g)$ for some $g \in \varnothing_{p}$ with $g \mathfrak{m}_{p} \subset I^{\prime}$, therefore $f I=f I^{\prime}+(f g) \subset I^{\prime}$ and $I \subset I^{\prime \prime}=\left(I^{\prime}: f\right)$, as wanted.

Corollary 4.11. If $(K, \mathbf{m})$ is a weighted (not necessarily unibranched) cluster such that $Z_{K, \mathbf{m}}$ imposes independent conditions to curves of degree $d$, then general curves in $E=E_{d+1}(K, \mathbf{m})$ go sharply through $(K, \mathbf{m})$. Furthermore, if $(K, \mathbf{m})$ is not a single point of multiplicity $d+1$, then a general curve in $E$ is irreducible.

Proof. Because of Lemma 4.10, $\mathrm{E}$ has no fixed part and $\mathrm{BP}(\mathrm{E})=(K, \mathbf{m})$. By Proposition 4.9 then, we only have to see that if $\mathrm{E}$ is composed of the curves of a pencil, then $\mathrm{BP}(\mathrm{E})$ is a point with multiplicity $d+1$. Let $\mathrm{E}$ be composed of $r$ curves of degree $k$ in a pencil. Then $r k=d+1$ and

$$
r=\ell_{d+1}(K, \mathbf{m})=d+2+\ell_{d}(K, \mathbf{m}) \geq d+1
$$

because $Z_{K, \mathbf{m}}$ imposes independent conditions to curves of degree $d$. Therefore, $r=d+1$ and $k=1$. A pencil of lines has a unique base point $p$ so curves in $\mathrm{E}$ are composed of $d+1$ lines through $p$ and $\mathrm{BP}(\mathrm{E})$ is as claimed.

The cluster of infinitely near singular points of a tacnode of order $t$ is a weighted unibranched cluster $K$ of $t$ free points with multiplicities $\mathbf{m}=\left(2^{t}\right)$. The scheme $Z_{K, \mathbf{m}}$ is called a tacnode scheme. The cluster of infinitely near singular points of a cusp of order $n$ is a weighted unibranched cluster $K$ of $n+2$ points, the last of which being satellite and the others free, with multiplicities $\mathbf{m}=\left(2^{n}, 1^{2}\right)$. We define an "extended" unibranched cluster $\left(K^{\prime}, \mathbf{m}^{\prime}\right)$ which has an additional free point taken with multiplicity one and call $Z_{K^{\prime}, \mathbf{m}^{\prime}}$ a cusp scheme of order $n$. When this last point $p_{n+3} \in K^{\prime}$ varies in $E_{n+2} \cong \mathbb{P}^{1}$ we obtain a flat family of cluster schemes, because of Lemma 3.6] In the special position of $p_{n+3}$ which makes it proximate to $p_{n+1}$, unloading gives $\delta\left(\mathbf{m}^{\prime}\right)=\left(2^{n+1}, 0,0\right)$ so any cusp scheme of order $n$ can be specialized to the tacnode scheme of order $n+1$ given by the free points $p_{1}, \ldots, p_{n+1}$.

Theorem 4.12. If

$$
\frac{d(d+1)}{6} \geq \sum_{i=1}^{\tau} t_{i}+\sum_{i=1}^{\nu}\left(n_{i}+1\right) \geq 6
$$



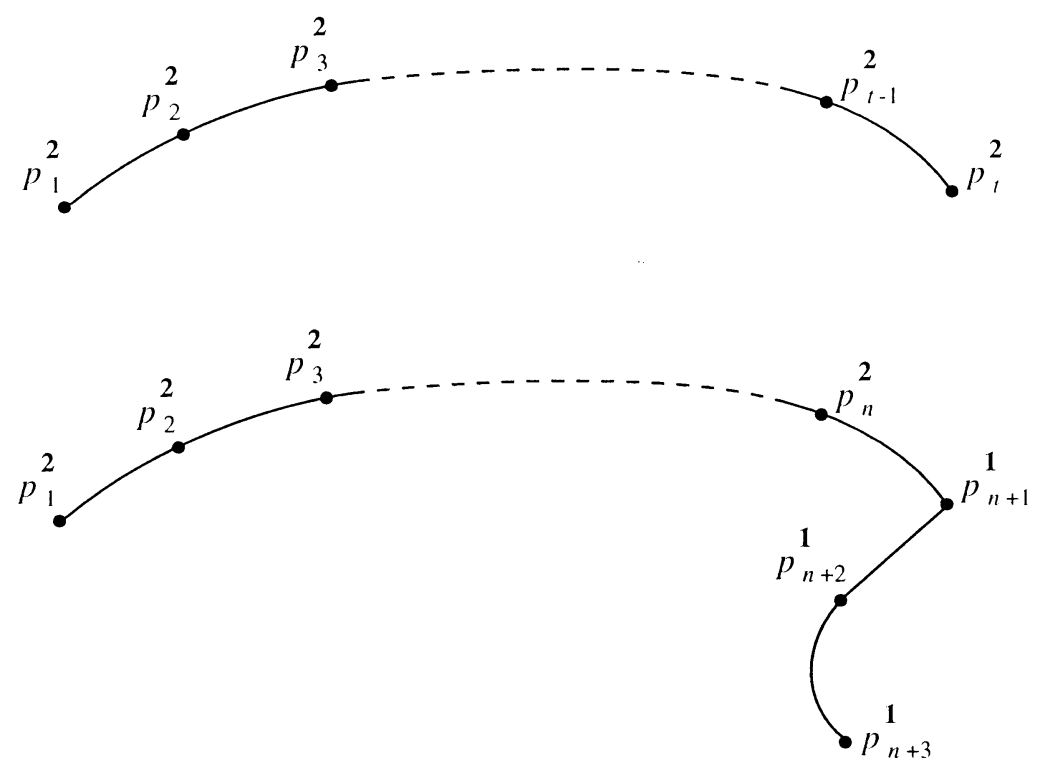

Figure 3. Enriques diagrams of a tacnode cluster and an extended cusp cluster.

then there exists a reduced irreducible curve of degree $d$ with $\tau$ tacnodes of orders $t_{1}, \ldots, t_{\tau}$ and $\nu$ cusps of orders $n_{1}, \ldots, n_{\nu}$ as its only singularities.

Proof. Consider a scheme

$$
Z=T_{1} \cup T_{2} \cup \cdots \cup T_{\tau} \cup N_{1} \cup N_{2} \cup \cdots \cup N_{\nu}
$$

where $T_{i}$ is a tacnode scheme of order $t_{i}$ supported at $p_{i} \in \mathbb{P}^{2}$ and $N_{i}$ is a cusp scheme of order $n_{i}$ supported at $q_{i}$, all of them having their points in general position. We claim that the linear system $\mathrm{E}_{d}(Z)$ of curves of degree $d$ containing $Z$ is nonempty and that a generic curve in it has a tacnode of order $t_{i}$ at $p_{i}$, a cusp of order $n_{i}$ at $q_{i}$ and no other singularities.

Specializing the cusp schemes $N_{i}$ to tacnode schemes $\bar{T}_{i}$ of order $n_{i}+1$ we obtain a scheme $\bar{Z}$ in the conditions of Theorem 4.8 therefore of maximal rank and length $3\left(\sum t_{i}+\sum\left(n_{i}+1\right)\right)$. So by semicontinuity $Z$ is also of maximal rank. The bound on $d$ assures that $Z$ imposes independent conditions to curves of degree $d-1$. So by 4.11 a general curve in $\mathrm{E}_{d}(Z)$ is irreducible and has no other singularities but the ones in $p_{i}, q_{i}$, which are tacnodes and cusps of the desired orders.

Note that in the hypotheses of Theorem 4.12 we ask that $\sum t_{i}+\sum\left(n_{i}+1\right) \geq 6$ in order to apply 4.8. In fact, the remarks we made after the proof of Theorem 4.8 prove that the result holds also for $\sum t_{i}+\sum\left(n_{i}+1\right)=3$, 4 . In case $\sum t_{i}+\sum\left(n_{i}+1\right)=$ 5 , an ad hoc reasoning can be used to prove the existence; however, for these small numbers of singularities the result is neither new nor significant, so we omit this.

We should like to point out that there are examples in [27] of curves $F_{k}$ with one tacnode or cusp, of degree lower than the one given by 4.12, Namely, they have 
degree $d=2 k+1$ and

$$
\left\{\begin{array}{l}
\text { a tacnode of order } \frac{2 k^{2}+3 k-1}{2} \text { if } 2 k^{2}+3 k-1 \text { is even, } \\
\text { a cusp of order } \frac{2 k^{2}+3 k-2}{2} \text { if } 2 k^{2}+3 k-1 \text { is odd. }
\end{array}\right.
$$

The author was informed by C. Lossen that he conjectures, after testing many particular cases, that these curves are irreducible and have no other singularity. He conjectures also that they do not satisfy the T-smoothness property (cf. [34]) which states that the variety of curves of degree $d$ with that singularity is smooth of the expected dimension at $F$. Curves with tacnodes and cusps whose points are in general position (as those given by 4.12) do satisfy the $\mathrm{T}$-smoothness property.

The reader may notice that after 4.11, any $h^{1}$-vanishing result for a class of cluster schemes can be exploited to obtain curves of low degree with the equisingularity type fixed by the clusters. In fact, the bounds obtained using 4.11 are (slightly) sharper than those obtained using the somewhat more complicated reasoning of [14. In particular, it is not difficult to extend Theorem 4.12 to curves with tacnodes, cusps, and one different singularity.

Proposition 4.13. Let $S$ be an equisingularity class whose cluster of infinitely near singular points is unibranched and consists of one point of multiplicity $m$ followed by $k$ free double points. Define $M=k+\sum_{i=1}^{\tau} t_{i}+\sum_{i=1}^{\nu}\left(n_{i}+1\right)+\max \left(0, \frac{m^{2}-4 m-6}{3}\right)$. If

$$
\frac{d(d+1)}{6}-\frac{m(m+1)}{6} \geq M \geq \frac{2}{3} m+1,
$$

then there exists a reduced irreducible curve of degree $d$ with one singularity of type $S, \tau$ tacnodes of orders $t_{1}, \ldots, t_{\tau}$ and $\nu$ cusps of orders $n_{1}, \ldots, n_{\nu}$ as its only singularities.

Proof. Analogous to the proof of 4.12

Note also that in the proof of Theorem 4.12 we proved that the union of general tacnode and cusp schemes has maximal rank, which is a case not included in Theorem 4.8. It is easy to see that many other cases may be equally treated using the same techniques. For example,

Proposition 4.14. The scheme of the cluster of infinitely near singular points of a $D_{k}$-singularity whose points are in general position has maximal rank, except in the two cases $k=6,7$.

Proof. The cluster of infinitely near singular points of a $D_{k}$-singularity, with $k$ even, is a weighted unibranched cluster with $k / 2-1$ free points with multiplicities $\mathbf{m}=\left(3,2^{k / 2-2}\right)$, so we are in a particular case of Theorem 4.8

If $k$ is odd, then the cluster of infinitely near singular points of $D_{k}$ is a weighted unibranched cluster with $r=(k+1) / 2$ points, the last of which being satellite and the others free (that is, $\left.K \in U_{r, r-2}\right)$, with multiplicities $\mathbf{m}=\left(3,2^{r-3}, 1^{2}\right)$. For simplicity, we assume $k \geq 15$ (or $r \geq 8$ ), as the cases with $k$ small require special care. Let $U \subset P_{r, r-2} \cap P_{3}$ be the open subset where only $p_{3}$ and $p_{r}$ are satellites (we specialize the third point to be proximate to the first). For $K \in U$, unloading gives $Z_{K, \mathbf{m}}=Z_{K, \mathbf{m}^{\prime}}$ with $\mathbf{m}^{\prime}=\left(4,2^{r-4}, 1,0,0\right)$, so the length of $Z_{K, \mathbf{m}}$ is the same for $K$ in $U$ or in $U_{r, r-2}$, therefore $U \subset\left(P_{r, r-2}\right)_{\mathbf{m}}$. As $U_{r, r-2}$ is dense in $\left(P_{r, r-2}\right)_{\mathbf{m}}$, by semicontinuity it is enough to see that $Z_{K, \mathbf{m}}=Z_{K, \mathbf{m}^{\prime}}$ has maximal rank for $K$ 
general in $U$ and the result follows from Theorem 4.8, because the multiplicity of the last point is now 0 so we can assume $K$ general in $U_{3}$.

\section{REFERENCES}

[1] Alexander, J. Hirschowitz, A., Interpolation on Jets J. Alg., 192 (1997), 412-417. MR 98i: 14052

[2] Barkats, D., Variétés des courbes planes à noeuds et à cusps, in: Algebraic Geometry, Peter E. Newstead, ed., Lect. Notes Pure App. Math. 200, M. Dekker 1988, pp. 25-35. MR 99j:14028

[3] Briançon, J., Description de Hilb ${ }^{n} \mathbb{C}\{x, y\}$, Inventiones Math. 41 (1977), 45-89. MR 56:15637

[4] Casas-Alvero, E., Infinitely near imposed singularities and singularities of polar curves, Math. Ann. 287 (1990), 429-454. MR 91h:14002

[5] Casas-Alvero, E., Singularities of plane curves, London Math. Soc. Lecture Notes Series, 276, Cambridge Univ. Press, Cambridge, 2000. CMP 2001:01

[6] Catalisano, M.V., Gimigliano, A. On curvilinear subschemes of $\mathbb{P}^{2}$ J. Pure App. Algebra 93 (1994), 1-14. MR 95f:14011

[7] Ciliberto, C. Miranda, R., Interpolation on Curvilinear Schemes, J. Alg. 203 (1998) 677-678. MR 99b:14047

[8] Ciliberto, C. Miranda, R., Degenerations of planar linear systems, Journal Reine Ang. Math. 501 (1998) 191-220. MR 2000m:14005

[9] Ciliberto, C. Miranda, R., Linear systems of plane curves with base points of equal multiplicity, (1998) Trans. Amer. Math. Soc. 352 (2000), 4037-4050. MR 2000m:14006

[10] Enriques, F., Chisini, O., Lezioni sulla teoria geometrica delle equazioni e delle funzioni algebriche, N. Zanichelli, Bologna 1915, Reprint, 1985. MR 90b:01106a

[11] Evain, L. Collisions de trois gros points sur une surface algébrique, thesis, Nice 1997.

[12] Evain, L., Une minoration du degré des courbes planes à singularités imposées, Preprint ENS Lyon, 212 (1997), 1-17.

[13] Fogarty, J., Algebraic families on an algebraic surface, Amer. J. Math 10 (1968) 511-521. MR 38:5778

[14] Greuel, G.M., Lossen, C., and Shustin, E., Plane curves of minimal degree with prescribed singularities, Inventiones Math. 133 (1998), 539-580. MR 99g:14035

[15] Greuel, G.M., Lossen, C., and Shustin, E., Castelnuovo function, zero-dimensional schemes and singular plane curves, J. Alg. Geom. 9 (2000), 663-710. CMP 2000:16

[16] Grothendieck, A., Dieudonné, J., Eléments de géométrie algébrique. IV, Inst. Hautes Etudes Sci. Publ. Math. 32 (1967).

[17] Harbourne, B., Complete linear systems on rational surfaces, Trans. Amer. Math. Soc. 289 (1985) 213-226. MR 86h:14030

[18] Harbourne, B., The geometry of rational surfaces and Hilbert functions of points in the plane, Can. Math. Soc. Conf. Proc. vol. 6 (1986) 95-111. MR 87k:14041

[19] Harbourne, B., Iterated blow-ups and moduli for rational surfaces, in: A. Holme and R. Speiser, eds., Algebraic Geometry Sundance 1986, LNM 1311, Springer 1988, pp. 101-117. MR 90b:14009

[20] Harbourne, B., Rational surfaces with $K^{2}>0$, Proc. Amer. Math. Soc. 124 (1996) 727-733. MR 96f:14045

[21] Harbourne, B., Anticanonical rational surfaces, Trans. Amer. Math. Soc. 349 (1997) 11911208. MR 97f:14007

[22] Hartshorne, B., Algebraic Geometry, GTM 52, Springer 1977.

[23] Hirschowitz, A., La méthode d'Horace pour l'interpolation à plusieurs variables, Manuscripta Math. 50 (1985), 337-388. MR 86j:14013

[24] Hirschowitz, A., Une conjecture pour la cohomologie des diviseurs sur les surfaces rationnelles generiques, J. Reine Angew. Math 397 (1989), 208-213. MR 90g:14021

[25] Iarrobino, A. Punctual Hilbert Schemes, Mem. Amer. Math. Soc. 10 (1977). MR 58:5667

[26] Kleiman, S.L., Multiple-point formulas I: Iteration, Acta Math. 147 (1981), 13-49. MR 83j:14006

[27] Lossen, C., New asymptotics for the existence of plane curves with prescribed singularities, Comm. in Algebra 27 (1999), 3263-3282. MR 2000e:14037

[28] Miranda, R., Linear systems of plane curves, Notices of the Amer. Math. Soc. 46, 2, (1999), 192-202. MR 99m:14012 
[29] Nobile, A., Villamayor, O., Equisingular stratifications associated to families of planar ideals, J. Alg. 193 (1997), 239-259. MR 98i:14005

[30] Paxia, G., On flat families of fat points, Proc. Amer. Math. Soc. 112 (1991), 19-23. MR 91m:14008

[31] Ran, Z., Curvilinear enumerative geometry, Acta Math. 155 (1985), 81-101. MR 86m:14040

[32] Ran, Z., Enumerative geometry of singular plane curves, Inventiones Math. 97 (1989), 447465. MR 90g:14039

[33] Roé, J., On the existence of plane curves with imposed multiple points, to appear in J. Pure App. Algebra

[34] Shustin, E., Smoothness of equisingular families of plane algebraic curves, Int. Math. Res. Notices (1997), 67-82. MR 97j:14031

[35] Zariski, O., Algebraic Surfaces, 2nd. suppl. ed., Ergebnisse 61, Springer-Verlag, 1971. MR 57:9695

[36] Zariski, O., Le problème des modules pour les branches planes, Hermann, 1986. MR 88a:14031

Departament D’Àlgebra i Geometria, Universitat de Barcelona, Gran Via, 585, E-08007, BARCELONA

E-mail address: jroevell@cerber.mat.ub.es 